\title{
Holographic complexity of Born-Infeld black holes
}

\author{
Kun Meng ${ }^{1,2, a}$ \\ ${ }^{1}$ School of Physics and Photoelectric Engineering, Weifang University, Weifang 261061, China \\ ${ }^{2}$ School of Physical Science and Technology, Tianjin Polytechnic University, Tianjin 300387, China
}

Received: 9 August 2019 / Accepted: 23 November 2019 / Published online: 3 December 2019

(C) The Author(s) 2019

\begin{abstract}
In this paper, according to CA duality, we study complexity growth of Born-Infeld (BI) black holes. As a comparison, we study action growth of dyonic black holes in Einstein-Maxwell gravity at the beginning. We study action growth of electric BI black holes in dRGT massive gravity, and find BI black holes in massive gravity complexify faster than the Einstein gravity counterparts. We study action growth of the purely electric and magnetic Einstein-BornInfeld (EBI) black holes in general dimensions and the dyonic EBI black holes in four-dimensions, and find the manners of action growth are different between electric and magnetic EBI black holes. In all the gravity systems we considered, we find action growth rates vanish for the purely magnetic black holes, which is unexpected. In order to ameliorate the situation, we add the boundary term of matter field to the action and discuss the outcomes of the addition.
\end{abstract}

\section{Introduction}

Holographic principle relates boundary CFT to bulk theory of gravity, through the correspondence one can study the problems of strong coupling CFT on the boundary through studying weak coupling gravity in the bulk. Remarkable progress has been made in applications of holographic principle in recent years, including applications of holography to study low energy QCD, hydrodynamics, condensed matter theory [1-5], etc.

Recently, the combination study of holography and quantum information shed light on understanding of quantum gravity. In the initial work [6], Maldacena and Susskind found any pair of entangled black holes are connected by some kind of Einstein-Rosen bridge, i.e., ER = EPR. However, the ER = EPR duality does not tell how it difficult to transmit information through Einstein-Rosen bridge. Therefore, the concept complexity was introduced. Complexity is the minimal num-

\footnotetext{
a e-mail: mengkun@tjpu.edu.cn
}

ber of simple gates needed to prepare a target state from a reference state. Complexity was originally conjectured to be proportional to the maximum volume of codimension-one surface bounded by the CFT slices, $\mathcal{C}=\frac{V}{G l}$, which is called CV duality [7-14]. The length scale $l$ is chosen according to situations. In order to eliminate the ambiguities in CV duality, CA duality was proposed $[15,16]$, which states that complexity is proportional to the action in Wheeler-DeWitt (WDW) patch, $\mathcal{C}=\frac{I}{\pi \hbar}$. CA duality does not involve any ambiguities encountered in CV duality but preserves all the nice features of $\mathrm{CV}$ duality. CA duality have passed the tests of shock wave and tensor network.

According to the definition, complexity growth rate is the speed of quantum computations. Considering black holes are the densest memories [17-19], it is conjectured that black holes are the fastest computers in nature. There exist a bound for the speed of quantum computation. Inspired by MargolusLevitin bound [20]

orthogonality time $\geq \frac{\pi \hbar}{2\langle E\rangle}$,

which gives the minimal time needed for a state evolving to an orthogonal state, Lloyd proposed a bound on the speed of computation [21]. Brown and collaborators generalized Lloyd's bound and conjectured that there exists a bound on the growth rate of complexity, which is

$\frac{d \mathcal{C}}{d t} \leq \frac{2 E}{\pi \hbar}$.

Calculations show that static neutral black holes saturate the bound.

It is natural to study complexity growth of black holes in different gravity systems to examine CA duality and Lloyd's bound. For the progresses in this subject please refer to $[16,22-42]$. In this paper, we intend to study complexity growth of BI black holes, since we are interested in the effects of nonlinearity of BI theory on complexity growth. The inner 
horizon of a BI black hole may turn into a curvature singularity due to perturbatively unstability [23], which implies a BI black hole may possess a single horizon [43-45]. It's interesting to study the differences in complexity between BI black holes with single horizon and AdS-Schwarzschild black holes, although the casual structures of them are identical. Since the magnetic black holes have been studied rarely, in this paper we will pay much attention to the magnetic black holes, and make a comparison of the effects between electric and magnetic charges on action growth. As we will see in the following, action growth of magnetic BI black holes exhibit some specific properties that are not found in the electric ones. We are also interested in studying action growth of BI black holes in massive gravity and study the effects of graviton mass.

Recently, the authors of Refs. [46-48] found that, action growth rates vanish for purely magnetic black holes in four dimensions. Which is unexpected since the expected latetime result $\frac{d I}{d t} \sim T S$ and electric-magnetic duality cannot be restored. Similar result was also found in this paper. In order to ameliorate the situation, a boundary term of matter field was proposed to be included to the action. In this paper, we add the boundary term of matter field proper to the gravity systems we considered and discuss the outcomes of the addition of the boundary term.

The paper is organized as, we study action growth of dyonic black holes in Einstein-Maxwell gravity in Sect. 2, BI black holes in massive gravity in Sect. 3, and EBI black holes in Sect. 4. In all the gravity systems we considered, we add the boundary term of matter field and discuss the outcomes of the addition. We summarize our calculations in the last section.

\section{Dyonic black holes of Einstein-Maxwell gravity}

In order to make a comparison between action growth of BI black holes and that of black holes in Einstein-Maxwell gravity, we first study dyonic black holes in Einstein-Maxwell gravity in this section. The action of the theory reads

$I=\frac{1}{16 \pi} \int d^{d} x \sqrt{-g}\left[R-2 \Lambda-\frac{1}{4} F_{\mu \nu} F^{\mu \nu}\right]$.

After taking variations of the metric and electromagnetic field, the field equations are given by

$G_{\mu \nu}+\Lambda g_{\mu \nu}=\frac{1}{2} F_{\mu \lambda} F_{\nu}{ }^{\lambda}-\frac{1}{8} F_{\alpha \beta} F^{\alpha \beta} g_{\mu \nu}$,

$\nabla_{\mu} F^{\mu \nu}=0$.

We take the metric and field strength ansatz for AdS planar black holes in $d=2 n+2$ dimensions as

$$
\begin{aligned}
d s^{2}= & -f(r) d t^{2}+\frac{d r^{2}}{f(r)} \\
& +r^{2}\left(d x_{1}^{2}+d x_{2}^{2}+\cdots+d x_{2 n-1}^{2}+d x_{2 n}^{2}\right), \\
F= & \Phi^{\prime}(r) d r \wedge d t+p\left(d x_{1} \wedge d x_{2}\right. \\
& \left.+\cdots+d x_{2 n-1} \wedge d x_{2 n}\right) .
\end{aligned}
$$

Solving equations of motion (e.o.m.) of the Maxwell field (4) we obtain

$\Phi(r)=\int_{r}^{\infty} \frac{q d r}{r^{2 n}}$.

Now we are able to solve the e.o.m. of metric (3), and obtain the dyonic black hole solution

$$
\begin{aligned}
f(r)= & -\frac{\mu}{r^{d-3}}-\frac{2 \Lambda}{(d-1)(d-2)} r^{2} \\
& +\frac{q^{2}}{2(d-2)(d-3) r^{2(d-3)}}-\frac{p^{2}}{4(d-5) r^{2}} .
\end{aligned}
$$

According to CA duality, complexity is proportional to action in WDW patch. Since WDW patch is in general nonsmooth, as shown by Fig. 1, we employ the method proposed in $[50,51]$ to calculate the action, which is given by

$$
\begin{aligned}
I_{\text {tot }}= & \int_{\mathcal{V}}\left(R-2 \Lambda+\mathcal{L}_{m a t}\right) \sqrt{-g} d V+2 \Sigma_{T_{i}} \int_{\partial \mathcal{V}_{T_{i}}} K d \Sigma \\
& +2 \Sigma_{S_{i}} \operatorname{sign}\left(S_{i}\right) \int_{\partial \mathcal{V}_{S_{i}}} K d \Sigma \\
& +2 \Sigma_{N_{i}} \operatorname{sign}\left(N_{i}\right) \int_{\partial \mathcal{V}_{N_{i}}} \kappa d S d \lambda \\
& +2 \Sigma_{j_{i}} \operatorname{sign}\left(j_{i}\right) \oint \eta_{j_{i}} d S \\
& +2 \Sigma_{m_{i}} \operatorname{sign}\left(m_{i}\right) \oint a_{m_{i}} d S .
\end{aligned}
$$

Where $S_{i}, T_{i}$ and $N_{i}$ labels spacelike, timelike and null boundary respectively. $K$ is the Gibbons-Hawking term. $\kappa$ measures the failure of $\lambda$ to be an affine parameter on the null generators. $\eta_{j_{i}}$ is the joint term between non-null hypersurfaces. $a_{m_{i}}$ is the joint term between null and other types of surfaces. The signatures $\operatorname{sign}\left(N_{i}\right), \operatorname{sign}\left(j_{i}\right), \operatorname{sign}\left(m_{i}\right)$ are determined through the requirement that the gravitational action is additive.

To proceed the calculations, it's convenient to introduce null coordinates

$d u \equiv d t+f^{-1} d r, \quad d v \equiv d t-f^{-1} d r$.

Under the null coordinates the metric becomes

$d s^{2}=-f d u^{2}+2 d u d r+r^{2} h_{i j} d x^{i} d x^{j}$, 
or

$d s^{2}=-f d v^{2}-2 d v d r+r^{2} h_{i j} d x^{i} d x^{j}$.

Since the dyonic black holes (7) possess both inner and outer horizons, we only need the right panel of Fig. 1 to calculate action growth. From the right panel of Fig. 1 we obtain the bulk contribution to $\delta I$

$I_{\mathcal{V}_{1}}=\frac{1}{16 \pi} \omega_{2}^{n} \int_{u}^{u+\delta t} d u \int_{r_{-}}^{r_{\max }} d r r^{d-2} \mathcal{L}_{\text {bulk }}$

$I_{\mathcal{V}_{2}}=\frac{1}{16 \pi} \omega_{2}^{n} \int_{v}^{v+\delta t} d v \int_{r_{+}}^{r_{\max }} d r r^{d-2} \mathcal{L}_{\text {bulk }}$

where $r_{\max }$ is the UV cutoff, and $\omega_{2} \equiv \int d x_{1} d x_{2}=\cdots=$ $\int d x_{2 n-1} d x_{2 n}$. With the e.o.m. (3), we have

$\mathcal{L}_{\text {bulk }}=\frac{4 \Lambda}{d-2}+\frac{q^{2}}{(d-2) r^{2(d-2)}}-\frac{p^{2}}{2 r^{4}}$.

Therefore, the bulk contribution to total action growth is

$$
\begin{aligned}
I_{\mathcal{V}_{1}}-I_{\mathcal{V}_{2}}= & \frac{1}{16 \pi} \omega_{2}^{n} \delta t\left[\frac{4 \Lambda r_{+}^{d-1}}{(d-1)(d-2)}-\frac{p^{2} r_{+}^{d-5}}{2(d-5)}\right. \\
& \left.-\frac{q^{2}}{(d-2)(d-3) r_{+}^{d-3}}\right]\left.\right|_{r_{-}} ^{r_{+}}
\end{aligned}
$$

As shown by the right panel of Fig. 1, there are four joints between null surfaces contributing to $\delta I$. Actions of the joints are given by

$$
\begin{aligned}
I_{\mathcal{B}^{\prime} \mathcal{B}} & =\frac{1}{16 \pi}\left[2 \oint_{\mathcal{B}^{\prime}} a d S-2 \oint_{\mathcal{B}} a d S\right] \\
& =\frac{1}{8 \pi} \omega_{2}^{n}\left[h\left(r_{\mathcal{B}^{\prime}}\right)-h\left(r_{\mathcal{B}}\right)\right],
\end{aligned}
$$

where $a=\ln \left(-\frac{1}{2} k \cdot \bar{k}\right)$, with $k$ being the null normal to the hypersurface $v=$ const and $\bar{k}$ being the null normal to the hypersurface $u=$ const. For the affinely parametrized expressions $k_{\alpha}=-c \partial_{\alpha} v$ and $\bar{k}_{\alpha}=\bar{c} \partial_{\alpha} u$, we have $a=-\ln (-f /(c \bar{c}))$, therefore $h(r)=-r^{d-2} \ln (-f /(c \bar{c}))$. Using $d r=-\frac{1}{2} f \delta t$, we obtain

$I_{\mathcal{B}^{\prime} \mathcal{B}}=\left.\frac{1}{16 \pi} \omega_{2}^{n} \delta t\left[r^{d-2} f^{\prime}+(d-2) r^{d-3} f \ln \left(\frac{-f}{c \bar{c}}\right)\right]\right|_{r=r_{\mathcal{B}}}$.

At late times, $r_{\mathcal{B}} \rightarrow r_{+}, r_{\mathcal{C}} \rightarrow r_{-}$, we have

$$
\begin{aligned}
I_{\mathcal{B}^{\prime} \mathcal{B}} & =\left.\frac{1}{16 \pi} \omega_{2}^{n} r^{d-2} f^{\prime}(r)\right|_{r_{+}} \delta t, \\
I_{\mathcal{C}^{\prime} \mathcal{C}} & =-\left.\frac{1}{16 \pi} \omega_{2}^{n} r^{d-2} f^{\prime}(r)\right|_{r_{-}} \delta t .
\end{aligned}
$$

The two terms in (17) together give rise to

$$
\begin{aligned}
I_{\mathcal{B}^{\prime} \mathcal{B}}+I_{\mathcal{C}^{\prime} \mathcal{C}}= & \frac{1}{16 \pi} \omega_{2}^{n} \delta t\left[-\frac{4 \Lambda r_{+}^{d-1}}{(d-1)(d-2)}\right. \\
& \left.+\frac{p^{2} r_{+}^{d-5}}{2(d-5)}-\frac{q^{2}}{(d-2) r_{+}^{d-3}}\right]\left.\right|_{r_{-}} ^{r_{+}}
\end{aligned}
$$

The total action growth is obtained by sum of all the contributions

$$
\begin{aligned}
\frac{\delta I}{\delta t} & =\frac{1}{16 \pi}\left[-\frac{q^{2}}{(d-3) r_{+}^{d-3}}+\frac{q^{2}}{(d-3) r_{-}^{d-3}}\right] \\
& =\left[\left(M-Q_{e} \Phi_{e}\right)_{+}-\left(M-Q_{e} \Phi_{e}\right)_{-}\right] .
\end{aligned}
$$

Where $Q_{e}=\frac{q}{16 \pi}, \Phi_{e}=\frac{q}{(d-3) r_{ \pm}^{d-3}}$ are electric charge and potential respectively. It can be seen that, similar to the fourdimensional case [46], magnetic charge does not contribute to action growth, which implies action growth rates vanish for purely magnetically charged black holes. In order to restore the late-time result $\frac{d I}{d t} \sim T S$ for purely magnetic black holes and electric-magnetic duality in four dimensions, the action of Einstein-Maxwell theory may be modified by a boundary term of Maxwell field.

The Maxwell boundary term which is considered to be included to the action reads [46]

$I_{\mu Q}=\frac{\gamma}{16 \pi} \int_{\partial \mathcal{M}} d \Sigma_{\mu} F^{\mu \nu} A_{\nu}$

This term does not affect the field equations but only alter the boundary conditions in the variational principle of Maxwell field. A well-posed variational principle requires Dirichlet boundary condition $\delta A_{a}=0$ for the original Maxwell action in (2), while after adding the boundary term (20), it requires Neumann boundary condition $n^{\mu} \partial_{\mu} \delta A_{a}=0$ for $\gamma=1$ and mixed boundary conditions for general $\gamma$. Addition of the boundary term (20) is natural when studying thermodynamics or Euclidean action, it produces the Legendre transformation from a grand canonical ensemble with fixed chemical potential to a canonical one with fixed charge. Using the field equations $\nabla_{\mu} F^{\mu \nu}=0$ and Stokes' theorem the boundary term (20) can be rewritten as

$I_{\mu Q}=\frac{\gamma}{32 \pi} \int_{\mathcal{M}} d^{d} x \sqrt{-g} F^{\mu \nu} F_{\mu \nu}$

Action growth for the dyonic black holes now becomes

$$
\begin{aligned}
\frac{\delta I}{\delta t}= & {\left[M-(1-\gamma) Q_{e} \Phi_{e}-\gamma Q_{m} \Phi_{m}\right]_{+} } \\
& -\left[M-(1-\gamma) Q_{e} \Phi_{e}-\gamma Q_{m} \Phi_{m}\right]_{-} .
\end{aligned}
$$



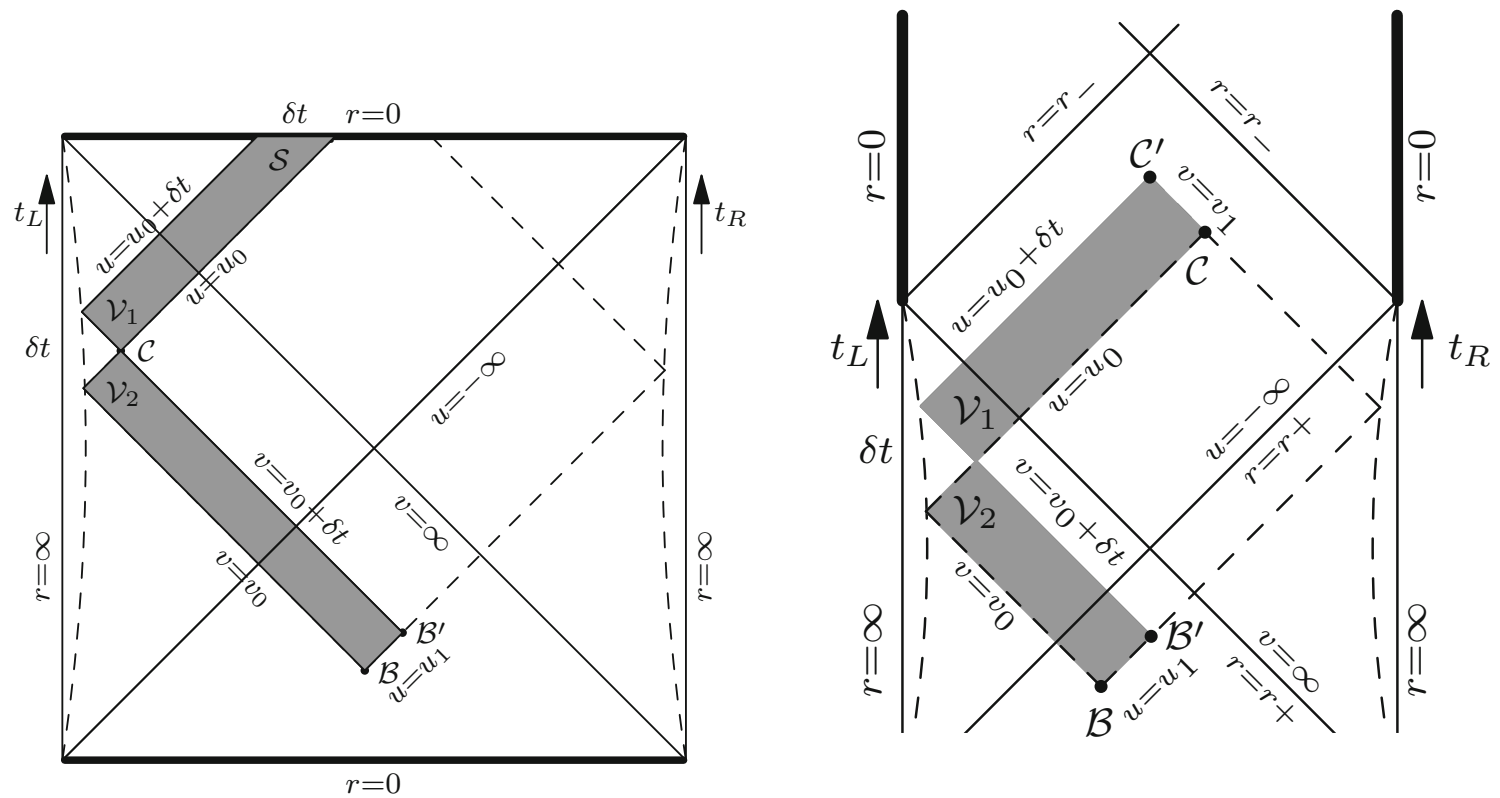

Fig. 1 Wheeler-DeWitt (WDW) patch of black holes. The left panel represents WDW patch of black holes with single horizon, the right panel represents WDW patch of black holes with double horizons

This result takes the identical form with that of the fourdimensional black hole [46]. One sees that, if we take $\gamma=$ $\frac{1}{2}$, then electric and magnetic charges contribute to action growth on equal footing, this agrees with electric-magnetic duality in four dimensions. If we take $\gamma=1$, contrary to the $\gamma=0$ case, only magnetic charge contributes to action growth, i.e., action growth rates vanish at late times for purely electrically charged black holes in this case.

\section{BI black holes in massive gravity}

In this section, we study action growth of purely electrically charged BI black holes in massive gravity and discuss the effects of graviton mass. The action of Einstein massive gravity is given by [49]

$$
\begin{aligned}
I & =\frac{1}{16 \pi} \int d^{d} x \sqrt{-g} \\
& \times\left[R-2 \Lambda+\mathcal{L}(\mathcal{F})+m^{2} \sum_{i=1}^{4} c_{i} \mathcal{U}_{i}(g, f)\right],
\end{aligned}
$$

where $f$ is a fixed symmetric rank-2 tensor. $c_{i}$ are constants and $\mathcal{U}_{i}$ are symmetric polynomials of the eigenvalues of matrix $\mathcal{K}_{v}^{\mu} \equiv \sqrt{g^{\mu \alpha} f_{\alpha \nu}}$

$$
\begin{aligned}
& \mathcal{U}_{1}=[\mathcal{K}], \quad \mathcal{U}_{2}=[\mathcal{K}]^{2}-\left[\mathcal{K}^{2}\right], \\
& \mathcal{U}_{3}=[\mathcal{K}]^{3}-3[\mathcal{K}]\left[\mathcal{K}^{2}\right]+2\left[\mathcal{K}^{3}\right], \\
& \mathcal{U}_{4}=[\mathcal{K}]^{4}-6\left[\mathcal{K}^{2}\right][\mathcal{K}]^{2}+8\left[\mathcal{K}^{3}\right][\mathcal{K}]+3\left[\mathcal{K}^{2}\right]^{2}-6\left[\mathcal{K}^{4}\right] .
\end{aligned}
$$

$\mathcal{L}(\mathcal{F})$ is the Lagrangian density of BI theory

$$
\mathcal{L}(\mathcal{F})=4 \beta^{2}\left(1-\sqrt{1+\frac{F^{\rho \sigma} F_{\rho \sigma}}{2 \beta^{2}}}\right) .
$$

Taking variations of the metric and electromagnetic field one obtains the field equations

$G_{\mu \nu}+\Lambda g_{\mu \nu}-\frac{1}{2} g_{\mu \nu} \mathcal{L}(\mathcal{F})-\frac{2 F_{\mu \lambda} F_{\nu}^{\lambda}}{\sqrt{1+\frac{F^{\rho \sigma} F_{\rho \sigma}}{2 \beta^{2}}}}+m^{2} \chi_{\mu \nu}=0$,

$\partial_{\mu}\left(\frac{\sqrt{-g} F^{\mu \nu}}{\sqrt{1+\frac{F^{\rho \sigma} F_{\rho \sigma}}{2 \beta^{2}}}}\right)=0$

where

$$
\begin{aligned}
\chi_{\mu \nu}= & -\frac{c_{1}}{2}\left(\mathcal{U}_{1} g_{\mu \nu}-\mathcal{K}_{\mu \nu}\right) \\
& -\frac{c_{2}}{2}\left(\mathcal{U}_{2} g_{\mu \nu}-2 \mathcal{U}_{1} \mathcal{K}_{\mu \nu}\right. \\
& \left.+2 \mathcal{K}_{\mu \nu}^{2}\right)-\frac{c_{3}}{2}\left(\mathcal{U}_{3} g_{\mu \nu}-3 \mathcal{U}_{2} \mathcal{K}_{\mu \nu}\right. \\
& \left.\times 6 \mathcal{U}_{1} \mathcal{K}_{\mu \nu}^{2}-6 \mathcal{K}_{\mu \nu}^{3}\right)-\frac{c_{4}}{2}\left(\mathcal{U}_{4} g_{\mu \nu}-4 \mathcal{U}_{3} \mathcal{K}_{\mu \nu}\right. \\
& \left.+12 \mathcal{U}_{2} \mathcal{K}_{\mu \nu}^{2}-24 \mathcal{U}_{1} \mathcal{K}_{\mu \nu}^{3}+24 \mathcal{K}_{\mu \nu}^{4}\right) .
\end{aligned}
$$

We take the static metric ansatz

$d s^{2}=-f(r) d t^{2}+\frac{d r^{2}}{f(r)}+r^{2} h_{i j} d x^{i} d x^{j}$, 
where $h_{i j} d x^{i} d x^{j}$ is the line element of codimension-two hypersurface with constant curvature. Using the reference metric

$f_{\mu \nu}=\operatorname{diag}\left(0,0, h_{i j}\right)$,

the $\mathcal{U}_{i}$ 's can be expressed as

$\mathcal{U}_{1}=\frac{d_{2}}{r}, \quad \mathcal{U}_{2}=\frac{d_{2} d_{3}}{r^{2}}, \quad \mathcal{U}_{3}=\frac{d_{2} d_{3} d_{4}}{r^{3}}$,

$\mathcal{U}_{4}=\frac{d_{2} d_{3} d_{4} d_{5}}{r^{4}}$

where $d_{i} \equiv d-i$ is introduced for convenience. Under the assumption of electrostatic potential $A_{\mu}=\Phi(r) \delta_{\mu}^{0}$, we solve the e.o.m of $A_{\mu}$ and find the only non-vanishing component of strength tensor is

$F_{t r}=\frac{\sqrt{d_{2} d_{3}} q}{r^{d_{2}} \sqrt{1+\frac{d_{2} d_{3} q^{2}}{\beta^{2} r^{2 d_{2}}}}}$

Substituting the above results into (26), one obtains the black hole solution

$$
\begin{aligned}
f(r)= & k-\frac{m_{0}}{r^{d_{3}}}+\frac{4 \beta^{2}-2 \Lambda}{d_{1} d_{2}} r^{2} \\
& -\frac{4 \beta^{2} r^{2}}{d_{1} d_{2}} \sqrt{1+\Gamma}+\frac{4 d_{2} q^{2}}{d_{1} r^{2 d_{3}}} \mathcal{H} \\
& +m^{2}\left(\frac{c_{1} r}{d_{2}}+c_{2}+\frac{d_{3} c_{3}}{r}+\frac{d_{3} d_{4} c_{4}}{r^{2}}\right),
\end{aligned}
$$

with

$\Gamma=\frac{d_{2} d_{3} q^{2}}{\beta^{2} r^{2 d_{2}}}, \quad \mathcal{H}={ }_{2} F_{1}\left[\frac{1}{2}, \frac{d_{3}}{2 d_{2}}, \frac{3 d-7}{2 d_{2}},-\Gamma\right]$.

The details of geometry and thermodynamics of the black hole (33) can be found in [49]. The black hole may possess single or double horizons. Let's first calculate action growth of the single-horizoned black hole. The left panel of Fig. 1 shows us the Penrose diagram of this type of black holes. From the panel it is easy to see that, the $\eta$ terms in (8) vanish because there are no joints between non-null hypersurfaces in WDW patch. It is natural to require all null segments to be affine parametrized, therefore all $\kappa$ terms in (8) vanish too. Considering time transition symmetry, the left contributions to $\delta I=I\left(t_{0}+\delta t\right)-I\left(t_{0}\right)$ are

$\delta I=I_{\mathcal{V}_{1}}-I_{\mathcal{V}_{2}}-2 \int_{\mathcal{S}} K d \Sigma+2 \oint_{\mathcal{B}^{\prime}} a d S-2 \oint_{\mathcal{B}} a d S$
With the e.o.m (26), we obtain the expression of bulk Lagrangian

$$
\begin{aligned}
\mathcal{L}_{\text {bulk }}= & \frac{4 \Lambda}{d_{2}}-\frac{8 \beta^{2}}{d_{2}}\left(1-\sqrt{1+\frac{d_{2} d_{3} q^{2}}{\beta^{2} r^{2} d_{2}}}\right) \\
& -\frac{m^{2}}{d_{2}}\left[\frac{d_{2} c_{1}}{r}-\frac{d_{2} d_{3} d_{4} c_{3}}{r^{3}}-2 \frac{d_{2} d_{3} d_{4} d_{5} c_{4}}{r^{4}}\right] .
\end{aligned}
$$

Thus action of the bulk region $\mathcal{V}_{1}$ is given by

$$
\begin{aligned}
I_{\mathcal{V}_{1}}= & \frac{1}{16 \pi} \Omega_{d_{2}} \int_{u}^{u+\delta t} d u \int_{\epsilon}^{\rho(u)} d r r^{d_{2}} \mathcal{L}_{\text {bulk }}, \\
= & \frac{1}{16 \pi} \Omega_{d_{2}} \delta t\left[\frac{4 \Lambda}{d_{1} d_{2}} r^{d_{1}}-2 F(r)\right. \\
& \left.-m^{2}\left(\frac{c_{1}}{d_{2}} r^{d_{2}}-c_{3} d_{3} r^{d_{4}}-2 c_{4} d_{3} d_{4} r^{d_{5}}\right)\right]\left.\right|_{\epsilon} ^{\rho(u)} .
\end{aligned}
$$

where $\Omega_{d_{2}}$ is the volume of the codimension-two hypersurface, $r=\rho(u)$ represents the $v=v_{0}+\delta t$ surface, and $F(r) \equiv \int d r r^{d_{2}} \frac{4 \beta^{2}}{d_{2}}\left(1-\sqrt{1+\frac{d_{2} d_{3} q^{2}}{\beta^{2} r^{2 d_{2}}}}\right)$ is introduced for convenience. Similarly, action of the bulk region $\mathcal{V}_{2}$ is given by

$$
\begin{aligned}
I_{\mathcal{V}_{2}}= & \frac{1}{16 \pi} \Omega_{d_{2}} \int_{v}^{v+\delta t} d v \int_{\rho_{1}(v)}^{\rho_{0}(v)} d r r^{d_{2}} \mathcal{L}_{\text {bulk }}, \\
= & \frac{1}{16 \pi} \Omega_{d_{2}} \delta t\left[\frac{4 \Lambda}{d_{1} d_{2}} r^{d_{1}}-2 F(r)\right. \\
& \left.-m^{2}\left(\frac{c_{1}}{d_{2}} r^{d_{2}}-c_{3} d_{3} r^{d_{4}}-2 c_{4} d_{3} d_{4} r^{d_{5}}\right)\right]\left.\right|_{\rho_{1}(v)} ^{\rho_{0}(v)},
\end{aligned}
$$

where $r=\rho_{0(1)}(v)$ represents the $u=u_{0(1)}$ surface. In the late-time limit, $\rho_{1}(v) \rightarrow r_{+}$, we have

$$
\begin{aligned}
I_{\mathcal{V}_{1}}-I_{\mathcal{V}_{2}}= & \frac{1}{16 \pi} \Omega_{d_{2}} \delta t\left[\frac{4 \Lambda}{d_{1} d_{2}} r_{+}^{d_{1}}-2 F\left(r_{+}\right)+2 F(0)\right. \\
& \left.-m^{2}\left(\frac{c_{1}}{d_{2}} r_{+}^{d_{2}}-c_{3} d_{3} r_{+}^{d_{4}}-2 c_{4} d_{3} d_{4} r_{+}^{d_{5}}\right)\right],
\end{aligned}
$$

where we have taken $\epsilon \rightarrow 0$. Action of the spacelike surface $\mathcal{S}$ is

$$
\begin{aligned}
I_{\mathcal{S}} & =-\frac{1}{8 \pi} \int K d \Sigma \\
& =\frac{1}{16 \pi} \Omega_{d_{2}} \delta t d_{1}\left[m_{0}-F(0)\right] .
\end{aligned}
$$

where we have used the expression $K=\frac{-1}{r^{d_{2}}} \frac{d}{d r}\left(r^{d_{2}} \sqrt{-f}\right)$ for extrinsic curvature and taken the $r \rightarrow 0$ limit. At late times, $r_{\mathcal{B}} \rightarrow r_{+}$, the joint terms are given by 


$$
\begin{aligned}
I_{\mathcal{B}^{\prime} \mathcal{B}}= & \frac{1}{16 \pi} \Omega_{d_{2}} \delta t\left[d_{3} m_{0}-\frac{4 \Lambda}{d_{1} d_{2}} r_{+}^{d_{1}}-d_{3} F\left(r_{+}\right)+r_{+} F^{\prime}\left(r_{+}\right)\right. \\
& \left.+m^{2}\left(\frac{c_{1}}{d_{2}} r_{+}^{d_{2}}-c_{3} d_{3} r_{+}^{d_{4}}-2 c_{4} d_{3} d_{4} r_{+}^{d_{5}}\right)\right]
\end{aligned}
$$

Collecting our calculations, adding up all the contributions to $\delta I(39),(40)$ and (41), we finally arrive at

$\frac{\delta I}{\delta t}=2 M-Q \Phi-C$,

where $M, Q, \Phi$ are respectively mass, electric charge and potential of the black hole

$$
\begin{aligned}
M & =\frac{d_{2} m_{0} \Omega_{d-2}}{16 \pi}, \quad Q=\frac{\sqrt{d_{2} d_{3}} q}{4 \pi}, \\
\Phi & =\sqrt{\frac{d_{2}}{d_{3}}} \frac{q}{r_{+}^{d_{3}}} 2 F_{1}\left[\frac{1}{2}, \frac{d_{3}}{2 d_{2}}, \frac{3 d-7}{2 d_{2}},-\frac{d_{2} d_{3} q^{2}}{\beta^{2} r_{+}^{2 d_{2}}}\right],
\end{aligned}
$$

and

$C=\frac{\beta^{2} d_{3}}{8 \pi d_{1} d_{2}}\left(d_{2} d_{3}\right)^{\frac{d_{1}}{2 d_{2}}}\left(\frac{q}{\beta}\right)^{\frac{d_{1}}{d_{2}}} \frac{\Gamma\left(1 /\left(2 d_{2}\right)\right) \Gamma\left(d_{3} /\left(2 d_{2}\right)\right)}{\Gamma(1 / 2)}$

Note that, action growth of the BI black holes in massive gravity (42) takes the identical form with that of EBI black holes [23]. However, graviton mass affects action growth through back-reaction on the geometry. The $m \rightarrow 0$ limit of (33) leads to black holes of EBI gravity

$$
\begin{aligned}
\hat{f}(r)= & k-\frac{m_{0}}{r^{d_{3}}}+\frac{4 \beta^{2}-2 \Lambda}{d_{1} d_{2}} r^{2} \\
& -\frac{4 \beta^{2} r^{2}}{d_{1} d_{2}} \sqrt{1+\Gamma}+\frac{4 d_{2} q^{2}}{d_{1} r^{2 d_{3}}} \mathcal{H} .
\end{aligned}
$$

When mass and electric charge of the black hole are fixed, we have $f(r)-\hat{f}(r)<0$ (given $c_{i}$ negative [52]) due to the graviton mass, which implies $r_{+}>\hat{r}_{+}\left(\hat{r}_{+}\right.$is the horizon of the EBI black hole (45)), therefore graviton mass leads BI black hole in massive gravity to complexitify faster than their Einstein gravity counterparts, just as the upper plot in Fig. 2 shows.

It is easy to note that, when $q \rightarrow 0$, action growth rate (42) reduces to the one of AdS-Schwarzschild black holes, for which Lloyd's bound is saturated (shown by the blue line of the upper plot in Fig. 2). Although the causal structure of single-horizoned BI black holes is identical with the one of AdS-Schwarzschild black holes, the manners of action growth differ between the two types of black holes due to the presence of electromagnetic field. Therefore, BI electromagnetic field slows down complexification of the black holes.
For black holes with inner and outer horizons, the causal structure of which is shown by the right panel of Fig. 1. Bulk contributions to $\delta I$ are given by

$$
\begin{aligned}
I_{\mathcal{V}_{1}}-I_{\mathcal{V}_{2}=} & \frac{1}{16 \pi} \Omega_{d_{2}} \delta t\left[\frac{4 \Lambda}{d_{1} d_{2}} r^{d_{1}}-2 F(r)\right. \\
& \left.-m^{2}\left(\frac{c_{1}}{d_{2}} r^{d_{2}}-c_{3} d_{3} r^{d_{4}}-2 c_{4} d_{3} d_{4} r^{d_{5}}\right)\right]\left.\right|_{r_{-}} ^{r_{+}}
\end{aligned}
$$

The joint terms of action are

$$
\begin{aligned}
I_{\mathcal{B}^{\prime} \mathcal{B}}+I_{\mathcal{C}^{\prime} \mathcal{C}}= & \frac{1}{16 \pi} \Omega_{d_{2}} \delta t\left[-\frac{4 \Lambda}{d_{1} d_{2}} r^{d_{1}}-d_{3} F(r)+r F^{\prime}(r)\right. \\
& \left.+m^{2}\left(\frac{c_{1}}{d_{2}} r^{d_{2}}-c_{3} d_{3} r^{d_{4}}-2 c_{4} d_{3} d_{4} r^{d_{5}}\right)\right]\left.\right|_{r_{-}} ^{r_{+}}
\end{aligned}
$$

The total variation of action is then given by the sum of Eqs. (46) and (47), which yields

$$
\frac{\delta I}{\delta t}=(M-Q \Phi)_{+}-(M-Q \Phi)_{-} .
$$

Just as the single horizon case, this result is formally identical with the one of EBI black holes. Similarly, we also have $f(r)-\hat{f}(r)<0$ for fixed mass and charge parameters, which implies $r_{-}<\hat{r}_{-}$and $r_{+}>\hat{r}_{+}\left(\hat{r}_{ \pm}\right.$are the inner and outer horizosn of the EBI black hole (45)), i.e., action growth rates of the double-horizoned BI black holes in Einstein massive gravity are superior to the ones of the Einstein gravity counterparts too, just as the lower two plots in Fig. 2 show us.

The lower left plot in Fig. 2 shows the case that there exists an extremal black hole for the selected parameters, the left most point $A$ represents the extremal black hole for which the inner and outer horizons merge. The difference between the line above $A$ and the line under $A$ is action growth rate of the black hole with double horizons. The lower right plot in Fig. 2 shows the case that no extremal black hole exists for the selected parameters. It is easy to see that action growth rates increase as $m$ increases.

Now, we add a boundary term of electromagnetic field to the total action

$$
\begin{aligned}
I_{\mu Q} & =\frac{\gamma}{16 \pi} \int d \Sigma_{\mu} \frac{4 F^{\mu \nu}}{\sqrt{1+\frac{F^{2}}{2 \beta^{2}}}} A_{\nu} \\
& =\frac{\gamma}{8 \pi} \int d^{d} x \sqrt{-g} \frac{1}{\sqrt{1+\frac{F^{2}}{2 \beta^{2}}}} F^{\mu \nu} F_{\mu \nu},
\end{aligned}
$$

which does not alter the field equations. In the second equality we have used Stokes' theorem and the field equations 


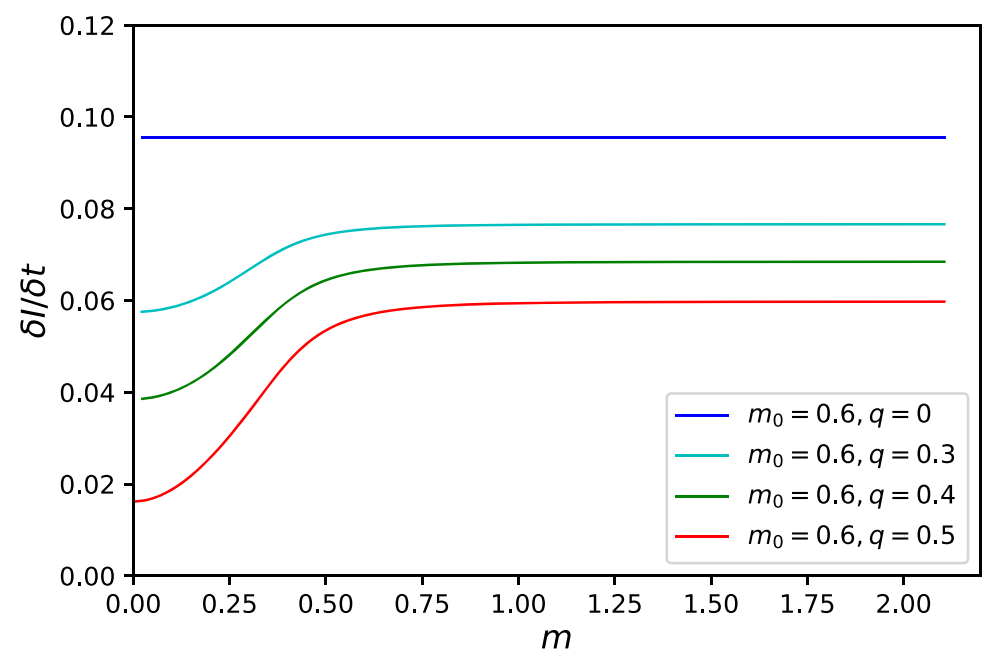

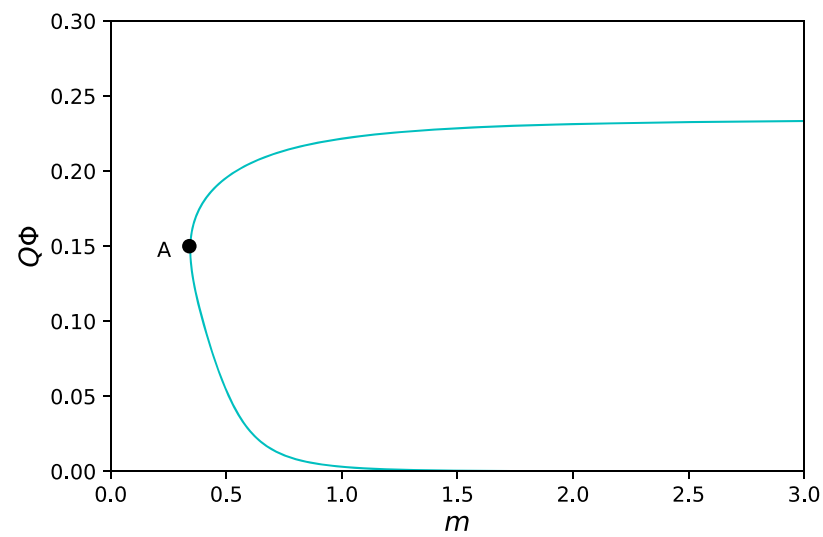

Fig. 2 The upper plot shows $\frac{\delta I}{\delta t}$ versus $m$ for black hole with single horizon. The parameters are fixed as $m_{0}=0.6, \Lambda=-1, \beta=$ $0.2, d=6, k=1, c_{1}=c_{2}=c_{3}=c_{4}=-1.5$. The lower two plots shows $Q \Phi$ versus $m$ for black holes with inner and outer horizons. For a fixed $m$, the difference between the upper line and

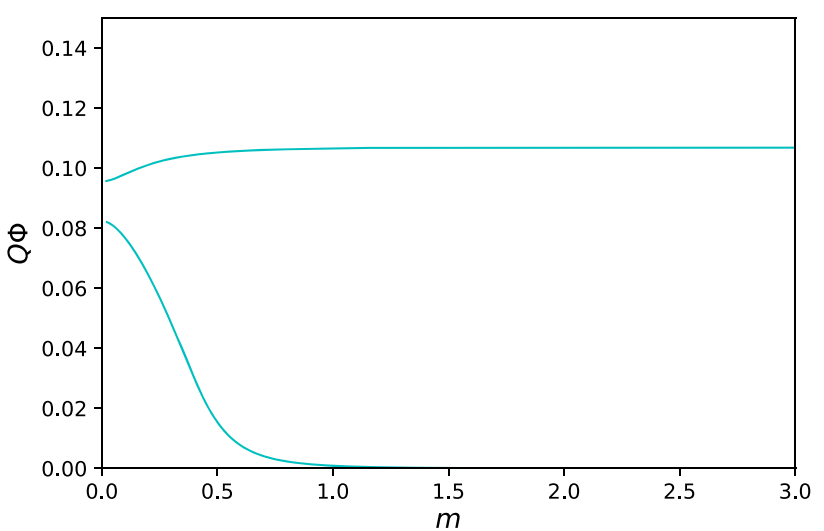

the lower line is action growth rate. The mass and electric charge parameters are fixed as $m_{0}=0.6, q=1.5$ (lower left plot) or $m_{0}=0.95, q=0.8$ (lower right plot), other parameters are fixed as $\Lambda=-1, \beta=0.2, d=6, k=1, c_{1}=c_{2}=c_{3}=c_{4}=-1.5$

$\frac{\delta I}{\delta t}=[M-(1-\gamma) Q \Phi]_{+}-[M-(1-\gamma) Q \Phi]_{-}$.

Note that, if we set $\gamma=1, Q \Phi$ does not appear in the expressions of action growth (50) and (52). In this case, for BI black holes with single horizon, electric charge affects action growth only through the constant $C_{1}$, for BI black holes with double horizons, action growth rates vanish. This agrees with the result obtained for dyonic black holes in Einstein-Maxwell gravity.

After addition of the BI boundary term, action growths versus $m$ are presented in Fig. 3 for different values of $\gamma$. The straight blue line on the upper plot in Fig. 3 still corresponds to the Lloyd's bound. The straight red line shows action growth of the black hole with single horizon for $\gamma=1$. The difference between the straight blue line and the straight red line is just $C_{1}$. The three points $A, B, C$ on the lower left becomes 


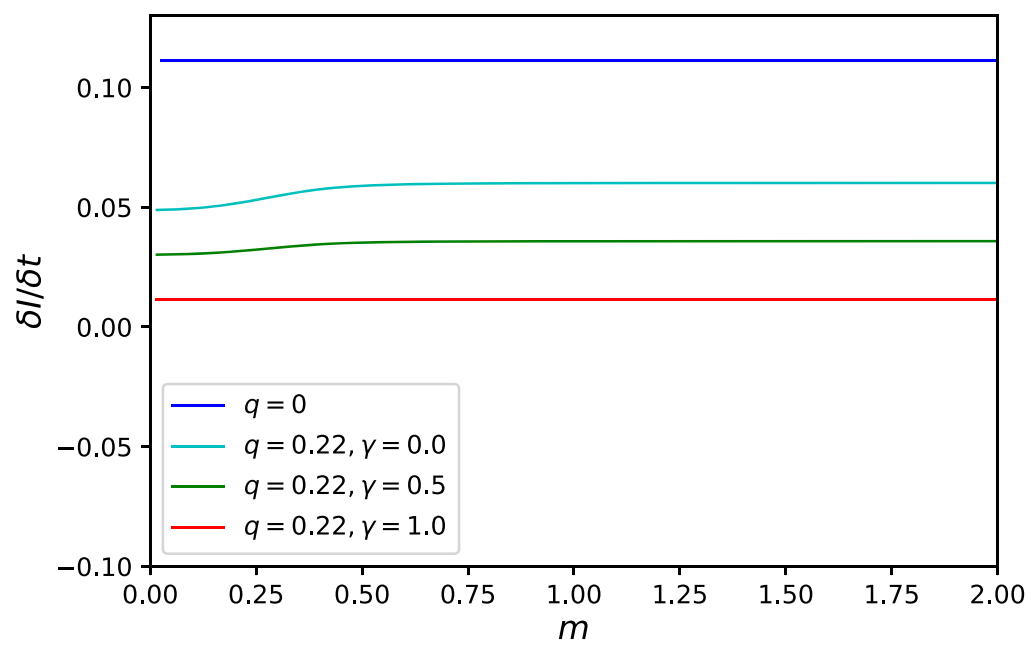

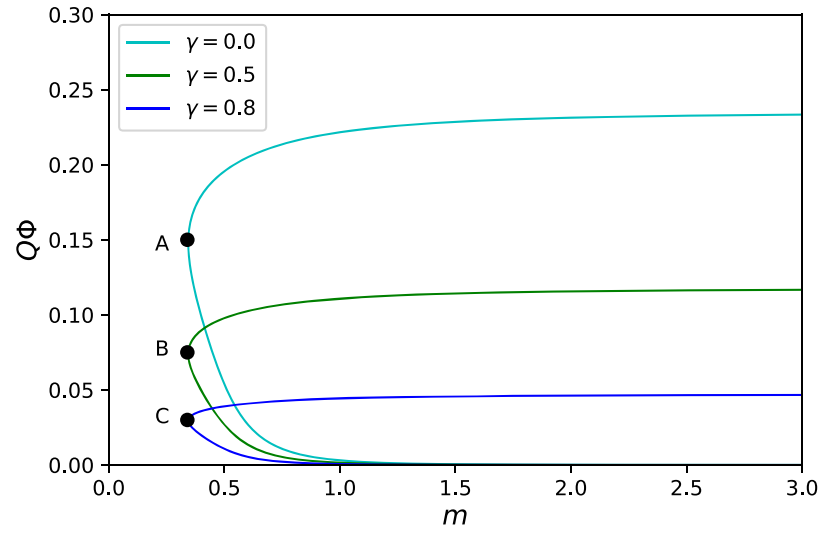

Fig. 3 The upper plot presents $\frac{\delta I}{\delta t}$ versus $m$ for the black holes with single horizon. The parameters are fixed as $m_{0}=0.7, \Lambda=-1, \beta=$ $0.2, d=6, k=1, c_{1}=c_{2}=c_{3}=c_{4}=-1.5$. The lower two plots present $Q \Phi$ versus $m$ for black holes with inner and outer horizons. For a fixed $m$, the difference between the upper line and the lower line is

plot in Fig. 3 still represent the extremal black holes. From the three plots in Fig. 3, one sees that, action growth rates decrease as $\gamma$ increases, and that action growth rates increase as $m$ increases.

\section{EBI black holes}

The Lagrangian (25) is only suitable for constructing BI black holes with electric charge. To construct black holes with both electric and magnetic charges, the Lagrangian (25) should be replaced with the general one [53]

$\mathcal{L}=-\beta^{2} \sqrt{-\operatorname{det}\left(g_{\mu \nu}+\frac{F_{\mu \nu}}{\beta}\right)}+\beta^{2} \sqrt{-\operatorname{det}\left(g_{\mu \nu}\right)}$.

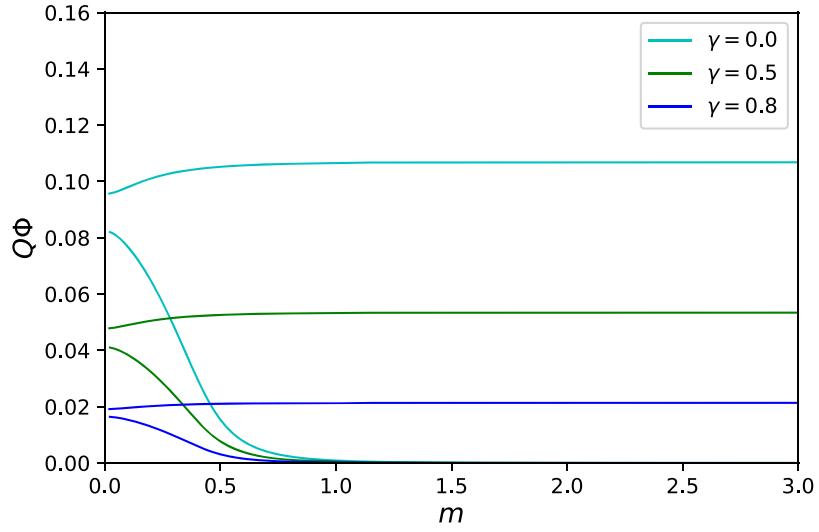

action growth rate. The mass and electric charge parameters are fixed as $m_{0}=0.6, q=1.5$ (lower left plot) or $m_{0}=0.95, q=0.8$ (lower right plot), other parameters are fixed as $\Lambda=-1, \beta=0.2, d=6, k=$ $1, c_{1}=c_{2}=c_{3}=c_{4}=-1.5$

In the large $\beta$ limit, (53) reduces to Maxwell theory. The Lagrangian of EBI theory is now given by

$\mathcal{L}=\sqrt{-g}\left(R-2 \Lambda_{0}\right)-\beta^{2} \sqrt{-\operatorname{det}\left(g_{\mu \nu}+\frac{F_{\mu \nu}}{\beta}\right)}$,

where $\Lambda_{0}=\Lambda-\beta^{2} / 2$ is the bare cosmological constant, and $\Lambda$ is the effective cosmological constant. Variation of the action of EBI theory give rise to field equations

$G^{\mu \nu}+\Lambda_{0} g^{\mu \nu}+\frac{\beta^{2}}{2} \frac{\sqrt{-h}}{\sqrt{-g}}\left(h^{-1}\right)^{(\mu \nu)}=0$,
$\nabla_{\mu}\left[\frac{\sqrt{-h}}{\sqrt{-g}} \beta\left(h^{-1}\right)^{[\mu \nu]}\right]=0$, 
where $h_{\mu \nu}=g_{\mu \nu}+F_{\mu \nu} / \beta, h \equiv \operatorname{det}\left(h_{\mu \nu}\right)$, and $\left(h^{-1}\right)^{\mu \nu}$ denotes the inverse of $h_{\mu \nu}$, i.e.

$\left(h^{-1}\right)^{\mu \rho} h_{\rho v}=\delta_{v}^{\mu}, \quad h_{v \rho}\left(h^{-1}\right)^{\rho \mu}=\delta_{v}^{\mu}$.

We still take the metric and strength ansatz given in Eq. (5). Solving e.o.m of $A_{\mu}$ (56), we have

$$
\Phi^{\prime}(r)=\frac{q}{\sqrt{\left(r^{4}+\frac{p^{2}}{\beta^{2}}\right)^{n}+\frac{q^{2}}{\beta^{2}}}},
$$

where " "' denotes derivative with respect to $r$. The Einstein equations (55) imply the dyonic black hole solution in general dimensions [53]

$f(r)=-\frac{\mu}{r^{2 n-1}}-\frac{\Lambda_{0}}{n(2 n+1)} r^{2}-\frac{\beta^{2}}{2 n r^{2 n-1}} G(r)$,

where $\mu$ is the mass parameter, and

$G(r) \equiv \int d r \sqrt{\left(r^{4}+\frac{p^{2}}{\beta^{2}}\right)^{n}+\frac{q^{2}}{\beta^{2}}}$

is introduced for convenience. The details of geometry and thermodynamics of the black hole (59) can be found in [53]. The integral (60) can not be integrated out for general $n$, let's consider the following special cases.

\subsection{Purely electric EBI black holes}

If we take the $p \rightarrow 0$ limit of the dyonic black holes (59), we obtain the purely electric EBI black holes. For electric EBI black holes with single horizon, with e.o.m (55) and the null coordinates introduced in (9), at late times the bulk contributions to action growth are given by

$$
\begin{aligned}
I_{\mathcal{V}_{1}}-I_{\mathcal{V}_{2}}= & \frac{1}{16 \pi} \omega_{2}^{n} \delta t\left[\frac{2 \Lambda_{0}}{n(2 n+1)} r_{+}^{2 n+1}\right. \\
& \left.+\frac{\beta^{2}}{n} \hat{G}\left(r_{+}\right)-\frac{\beta^{2}}{n} \hat{G}(0)\right] .
\end{aligned}
$$

where $\hat{G}(r) \equiv \int d r \sqrt{r^{4 n}+\frac{q^{2}}{\beta^{2}}}$ is $G(r)$ in (60) with the magnetic charge parameter $p=0$. Action of the $r=0$ surface is

$$
\begin{aligned}
I_{\mathcal{S}}= & \frac{1}{16 \pi} \omega_{2}^{n} \delta t[(2 n+1) \mu \\
& \left.-q^{\frac{2 n+1}{2 n}} \beta^{\frac{2 n-1}{2 n}} \frac{\Gamma(1 / 2-1 /(4 n)) \Gamma(1+1 /(4 n))}{\Gamma(1 / 2)}\right] .
\end{aligned}
$$

Joint terms in the action are given by

$$
\begin{aligned}
I_{\mathcal{B}^{\prime} \mathcal{B}}= & \frac{\omega_{2}^{n} \delta t}{16 \pi}\left[(2 n-1) \mu-\frac{2 \Lambda_{0}}{n(2 n+1)} r_{+}^{2 n+1}\right. \\
& \left.+\frac{2 n-1}{2 n} \beta^{2} \hat{G}\left(r_{+}\right)-\frac{\beta^{2} r_{+}}{2 n} \sqrt{r_{+}^{4 n}+\frac{q^{2}}{\beta^{2}}}\right] .
\end{aligned}
$$

By sum of Eqs. (61), (62) and (63) we have the action growth rate

$$
\begin{aligned}
\frac{\delta I}{\delta t}= & \frac{\omega_{2}^{n}}{16 \pi}\left[4 n \mu+\frac{2 n+1}{2 n} \beta^{2} \hat{G}\left(r_{+}\right)\right. \\
& \left.-\frac{\beta^{2} r_{+}}{2 n} \sqrt{r_{+}^{4 n}+\frac{q^{2}}{\beta^{2}}}-\frac{\beta^{2}}{n} \hat{G}(0)\right] \\
= & 2 M-Q_{e} \Phi_{e}-\hat{C}
\end{aligned}
$$

with

$M=\frac{n \omega_{2}^{n}}{8 \pi} \mu, \quad Q_{e}=\frac{q}{16 \pi} \omega_{2}^{n}, \quad \Phi_{e}=\int_{r_{+}}^{\infty} \frac{q d r}{\sqrt{r^{4 n}+\frac{q^{2}}{\beta^{2}}}}$

are mass, electric charge and potential respectively, and the constant

$\hat{C}=\frac{(2 n-1) \omega_{2}^{n} q^{\frac{2 n+1}{2 n}} \beta^{\frac{2 n-1}{2 n}}}{16 \pi(2 n+1)} \frac{\Gamma(1 / 2-1 /(4 n)) \Gamma(1+1 /(4 n))}{\Gamma(1 / 2)}$.

We see that, action growth of the electric EBI black holes is formally identical with that of the massive gravity counterparts. BI electromagnetic field does not change casual structure of the spacetime compared to AdS-Schwarzschild black holes, but it affects the manner of action growth, which leads Lloyd's bound to be unsaturated.

For electric EBI black holes with double horizons, the bulk contributions to $\delta I$ are

$$
I_{\mathcal{V}_{1}}-I_{\mathcal{V}_{2}}=\left.\frac{1}{16 \pi} \omega_{2}^{n} \delta t\left[\frac{2 \Lambda_{0}}{n(2 n+1)} r^{2 n+1}+\frac{\beta^{2}}{n} \hat{G}(r)\right]\right|_{r_{-}} ^{r_{+}}
$$

The joint terms of action are given by

$$
\begin{aligned}
I_{\mathcal{B}^{\prime} \mathcal{B}}+I_{\mathcal{C}^{\prime} \mathcal{C}}= & \frac{\omega_{2}^{n} \delta t}{16 \pi}\left[(2 n-1) \mu-\frac{2 \Lambda_{0}}{n(2 n+1)} r^{2 n+1}\right. \\
& \left.+\frac{2 n-1}{2 n} \beta^{2} \hat{G}(r)-\frac{\beta^{2} r}{2 n} \sqrt{r^{4 n}+\frac{q^{2}}{\beta^{2}}}\right]\left.\right|_{r_{-}} ^{r_{+}}
\end{aligned}
$$



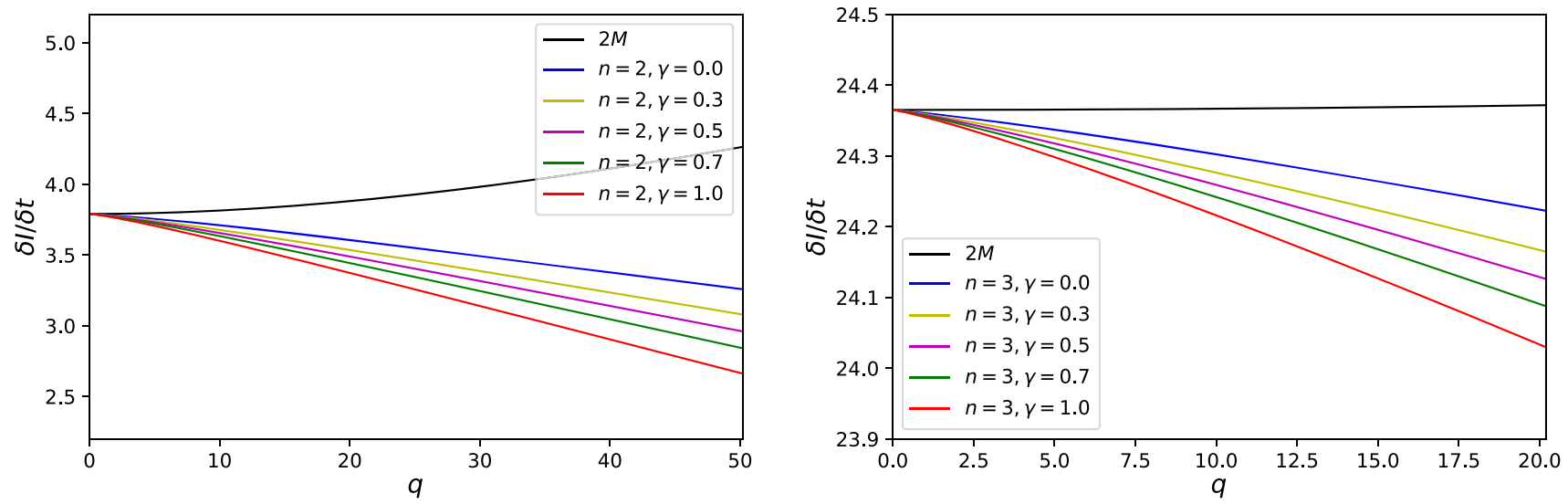

Fig. $4 \frac{\delta I}{\delta t}$ versus $q$ for the purely electric black holes with single horizon. The black line on each plot is the $2 M$ line. The parameters are fixed as $\beta=0.2, r_{+}=3, \Lambda=-1$

Combining (67) and (68) we have the total action growth rate

$$
\frac{\delta I}{\delta t}=\left(M-Q_{e} \Phi_{e}\right)_{+}-\left(M-Q_{e} \Phi_{e}\right)_{-} .
$$

In this case, action growth of electric EBI black holes takes the identical form with that of the AdS-RN black holes, however, electromagnetic field affects action growth through the nonlinearity of BI theory.

We add a boundary term of electromagnetic field to the action

$$
\begin{aligned}
I_{\mu Q} & =\frac{\gamma}{16 \pi} \int d \Sigma_{\mu} \beta \frac{\sqrt{-h}}{\sqrt{-g}}\left(h^{-1}\right)^{[\nu \mu]} A_{\nu} \\
& =\frac{\gamma}{32 \pi} \int d^{d} x \beta \sqrt{-h}\left(h^{-1}\right)^{[\nu \mu]} F_{\mu \nu},
\end{aligned}
$$

which does not affect the field equations. The field equations $\nabla_{\mu}\left(\frac{\sqrt{-h}}{\sqrt{-g}}\left(h^{-1}\right)^{[\nu \mu]}\right)=0$ and Stoke's theorem have been used in the second equality. For electric EBI black holes with single horizon, action growth becomes

$$
\frac{\delta I}{\delta t}=2 M-(1-\gamma) Q_{e} \Phi_{e}-\hat{C}_{1},
$$

with

$$
\begin{aligned}
\hat{C}_{1}= & \left(\frac{2 n-1}{2 n+1}+\gamma\right) \frac{\omega_{2}^{n} q^{\frac{2 n+1}{2 n}} \beta^{\frac{2 n-1}{2 n}}}{16 \pi} \\
& \times \frac{\Gamma(1 / 2-1 /(4 n)) \Gamma(1+1 /(4 n))}{\Gamma(1 / 2)} .
\end{aligned}
$$

Figure 4 shows us $\frac{\delta I}{\delta t}$ versus $q$. Since it is difficult to work out $q$ as $q\left(r_{+}\right)$, and subsequently $\frac{\delta I}{\delta t}$ as $\frac{\delta I}{\delta t}\left(r_{+}\right)$, from (59) for fixed $\mu$, we fix $r_{+}$, then $M$ and $\frac{\delta I}{\delta t}$ are functions of $q$. The black line on each plot in Fig. 4 is the $2 M$ line. From Fig. 4, one sees that action growth rates decrease as $q$ or $\gamma$ increases, Lloyd's bound is satisfied. Note that $\frac{\delta I}{\delta t}$ will not be zero as $q$ increases, since for certain value of the black hole mass $M, q$ can't be arbitrarily large to ensure the existence of the black hole horizon.

For electric EBI black holes with double horizons, action growth becomes

$$
\frac{\delta I}{\delta t}=\left[M-(1-\gamma) Q_{e} \Phi_{e}\right]_{+}-\left[M-(1-\gamma) Q_{e} \Phi_{e}\right]_{-} .
$$

As we will see in the following, action growth of the electric EBI black holes with $\gamma=1$ is in the same manner as that of the magnetic EBI black holes with $\gamma=0$, and vice versa.

\subsection{Pure magnetic EBI black holes}

In this subsection, we calculate action growth of purely magnetic EBI black hole in general dimensions and make a comparison between the effects of electric and magnetic charges on action growth.

For magnetic EBI black holes with single horizon, with e.o.m (55) and the null coordinates, we obtain the bulk contributions to $\delta I$

$$
\begin{aligned}
I_{\mathcal{V}_{1}}-I_{\mathcal{V}_{2}=} & \frac{1}{16 \pi} \omega_{2}^{n} \delta t\left[\frac{2 \Lambda_{0}}{n(2 n+1)} r^{2 n+1}\right. \\
& \left.+\frac{\beta^{2}}{n} \bar{G}(r)-p^{2} \bar{H}(r)\right]\left.\right|_{\epsilon} ^{r_{+}}, \\
= & \frac{1}{16 \pi} \omega_{2}^{n} \delta t\left[\frac{2 \Lambda_{0}}{n(2 n+1)} r_{+}^{2 n+1}+\frac{\beta^{2}}{n} \bar{G}\left(r_{+}\right)\right. \\
& \left.-p^{2} \bar{H}\left(r_{+}\right)-\frac{\beta^{2}}{n} \bar{G}(0)+p^{2} \bar{H}(0)\right] .
\end{aligned}
$$

where $\bar{G}(r) \equiv \int d r\left(r^{4}+\frac{p^{2}}{\beta^{2}}\right)^{n / 2}, \quad \bar{H}(r) \equiv \int d r$ $\left(r^{4}+\frac{p^{2}}{\beta^{2}}\right)^{n / 2-1}$, and we have taken the $\epsilon \rightarrow 0$ limit in the second equality. Action of the spacelike surface is given by 


$$
\begin{aligned}
I_{\mathcal{S}}= & \frac{1}{16 \pi} \omega_{2}^{n} \delta t[(2 n+1) \mu \\
& \left.+\frac{p^{n+1 / 2}}{2 n \beta^{n-3 / 2}} \frac{\Gamma(1 / 4) \Gamma(3 / 4-n / 2)}{\Gamma(-n / 2)}\right] .
\end{aligned}
$$

The joint terms in action are

$$
\begin{aligned}
I_{\mathcal{B}^{\prime} \mathcal{B}}= & \frac{\omega_{2}^{n} \delta t}{16 \pi}\left[(2 n-1) \mu-\frac{2 \Lambda_{0}}{n(2 n+1)} r_{+}^{2 n+1}\right. \\
& \left.+\frac{2 n-1}{2 n} \beta^{2} \bar{G}\left(r_{+}\right)-\frac{\beta^{2} r_{+}}{2 n} \sqrt{\left(r_{+}^{4}+p^{2} / \beta^{2}\right)^{n}}\right] .
\end{aligned}
$$

We have the total action growth by sum of Eqs. (74), (75) and (76)

$$
\begin{aligned}
\frac{\delta I}{\delta t}= & \frac{\omega_{2}^{n}}{16 \pi}\left[4 n \mu+\frac{2 n+1}{2 n} \beta^{2} \bar{G}\left(r_{+}\right)\right. \\
& -\frac{\beta^{2} r_{+}}{2 n} \sqrt{\left(r_{+}^{4}+p^{2} / \beta^{2}\right)^{n}} \\
& \left.-p^{2} \bar{H}\left(r_{+}\right)-\frac{\beta^{2}}{n} \bar{G}(0)+p^{2} \bar{H}(0)\right] \\
= & 2 M-\bar{C},
\end{aligned}
$$

with

$M=\frac{n \omega_{2}^{n}}{8 \pi} \mu$,

and

$\bar{C}=-\frac{\omega_{2}^{n} p^{n+1 / 2}}{8 \pi(2 n+1) \beta^{n-3 / 2}} \frac{\Gamma(1 / 4) \Gamma(3 / 4-n / 2)}{\Gamma(-n / 2)}$.

Note that, if we don't consider the BI boundary term, action growth of magnetic EBI black holes with single horizon depends only on mass and some constant involving $p$, it does not depend on the product of magnetic charge and potential $Q_{m} \Phi_{m}$. This differs from the purely electric case (64), where the action growth depends on mass, some constant and the product $Q_{e} \Phi_{e}$. By comparison of the first line of (77) and the first line of (64), we see that, for electric EBI black holes, the term corresponding to $\bar{H}\left(r_{+}\right)$disappears since $p$ vanishes, therefore the term $Q_{e} \Phi_{e}$ in action growth remains. The calculation details of simplifying Eq. (77) from the first line to the second line can be found in the appendix.

It's interesting to note that, $\bar{C}$ is a positive constant for $n$ to be odd, while $\bar{C}$ vanishes for $n$ to be even. For odd $n$, according to CA duality we have $\frac{d \mathcal{C}}{d t}<\frac{2 M}{\pi \hbar}$, Lloyd's bound is satisfied. For even $n$, we have $\frac{d \mathcal{C}}{d t}=\frac{2 M}{\pi \hbar}$, Lloyd's bound is saturated. Therefore, if we don't consider the boundary term of electromagnetic field, action growth of magnetic EBI black holes is in the same manner as the one of AdS-Schwarzschild black holes in some dimensions. In this case, magnetic charge affects action growth through back-reaction on the geometry.

For magnetic EBI black holes with double horizons, the bulk contributions to $\delta I$ are

$$
\begin{aligned}
I_{\mathcal{V}_{1}}-I_{\mathcal{V}_{2}}= & \frac{1}{16 \pi} \omega_{2}^{n} \delta t\left[\frac{2 \Lambda_{0}}{n(2 n+1)} r^{2 n+1}\right. \\
& \left.+\frac{\beta^{2}}{n} \bar{G}(r)-p^{2} \bar{H}(r)\right]\left.\right|_{r_{-}} ^{r_{+}} .
\end{aligned}
$$

The joint terms in action are

$$
\begin{gathered}
I_{\mathcal{B}^{\prime} \mathcal{B}}+I_{\mathcal{C}^{\prime} \mathcal{C}}=\frac{\omega_{2}^{n} \delta t}{16 \pi}\left[(2 n-1) \mu-\frac{2 \Lambda_{0}}{n(2 n+1)} r^{2 n+1}\right. \\
\left.+\frac{2 n-1}{2 n} \beta^{2} \bar{G}(r)-\frac{\beta^{2} r}{2 n} \sqrt{\left(r^{4}+p^{2} / \beta^{2}\right)^{n}}\right]\left.\right|_{r_{-}} ^{r_{+}}
\end{gathered}
$$

Combining (80) and (81) we find that

$\frac{\delta I}{\delta t}=0$.

This result agrees with that of the magnetic black holes in Einstein-Maxwell theory. Although action growth rates do not vanish for the magnetic EBI black holes with single horizon, action growth rates vanish for the magnetic black holes with double horizons both in Einstein-Maxwell gravity and in EBI gravity.

We now add the boundary term of electromagnetic field (70) to the action. For magnetic EBI black holes with single horizon, action growth becomes

$\frac{\delta I}{\delta t}=2 M-\gamma Q_{m} \Phi_{m}-\bar{C}_{1}$,

with $Q_{m}=\frac{n p}{16 \pi} \omega_{2}, \quad \Phi_{m}=\frac{\partial M}{\partial Q_{m}}$ are magnetic charge and potential respectively, and

$\bar{C}_{1}=-\frac{\omega_{2}^{n} p^{n+1 / 2}}{8 \pi \beta^{n-3 / 2}}\left(\frac{1}{2 n+1}-\frac{\gamma}{4}\right) \frac{\Gamma(1 / 4) \Gamma(3 / 4-n / 2)}{\Gamma(-n / 2)}$.

After the addition of the BI boundary term, we see that, magnetic charge affects action growth in a similar manner to electric charge. Here, $\bar{C}_{1}$ also vanishes for even $n$. Therefore, in the dimensions with even $n$, action growth of magnetic EBI black holes takes the specific form $\frac{\delta I}{\delta t}=2 M-\gamma Q_{m} \Phi_{m}$.

Figure 5 shows us $\frac{\delta I}{\delta t}$ versus $p$. Similar to the electric case, we fix $r_{+}$, the black line on each plot in Fig. 5 is the $2 M$ line. On the left two plots, the black lines coincide with the red lines, this is because $\bar{C}_{1}$ in (84) vanishes for even $n$. One sees from the figure that, Lloyd's bound may be violated for some values of $\gamma$. Therefore, the addition of matter field boundary term to action may lead to violation of 

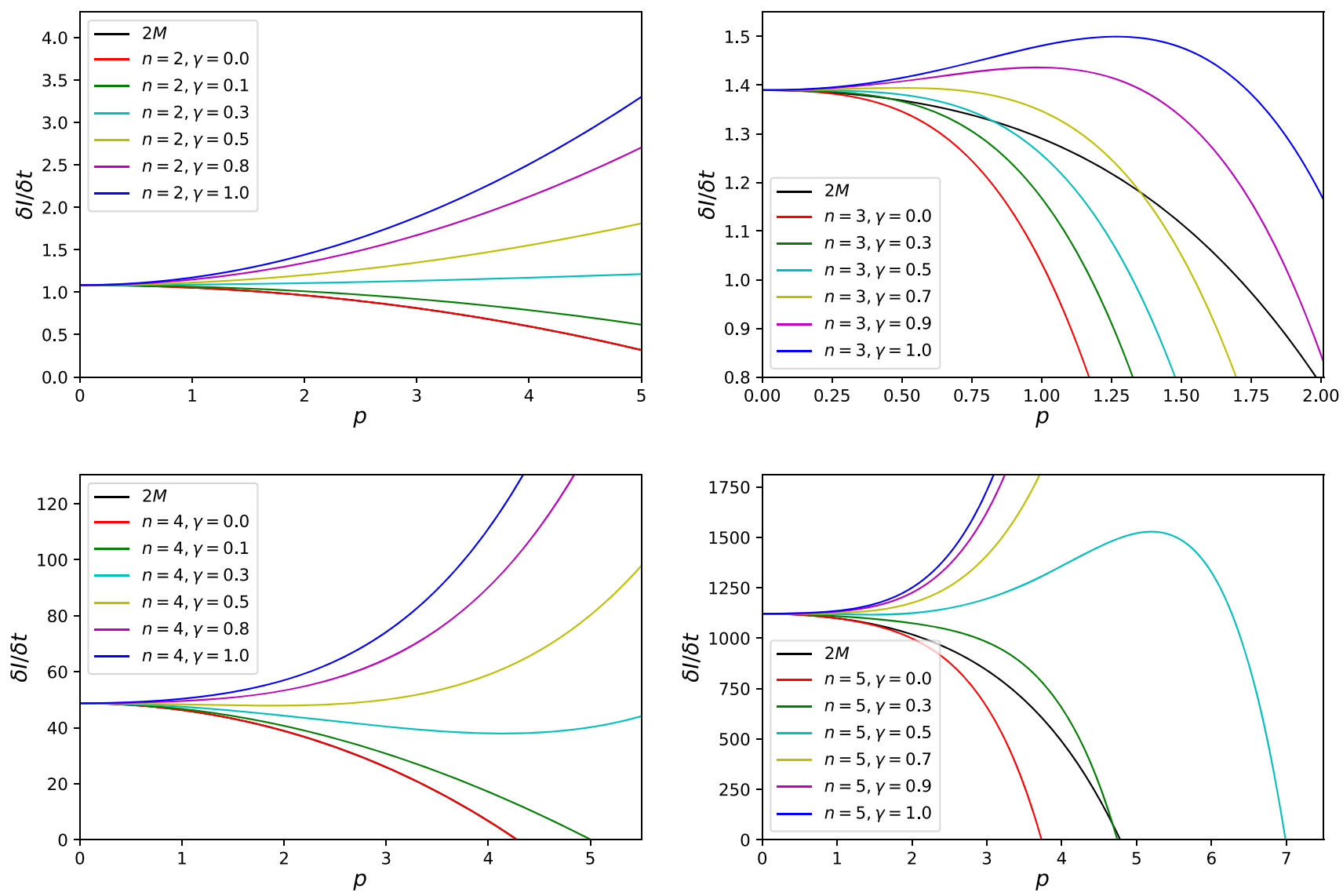

Fig. $5 \frac{\delta I}{\delta t}$ versus $p$ for the purely magnetic black holes with single horizon. The black line on each plot is the $2 M$ line. For the $n=2$ and $n=4$ cases, the black lines coincide with the red lines. The parameters

Lloyd's bound. The late-time violations of Lloyd's bound have also been found in Einstein-dilaton system, in EinsteinMaxwell-Dilaton system [26,36], etc. Note also that, $\frac{\delta I}{\delta t}$ will not be zero as $p$ increases, since $p$ can't be arbitrarily large for certain $M$ to ensure the existence of black hole horizon.

For magnetic BI black holes with double horizons, action growth becomes

$\frac{\delta I}{\delta t}=\left[\left(M-\gamma Q_{m} \Phi_{m}\right)\right]_{+}-\left[\left(M-\gamma Q_{m} \Phi_{m}\right)\right]_{-}$.

Now, the rate of action growth does not vanish due to addition of the BI boundary term. If we set $\gamma=1$, action growth of magnetic EBI black holes is formally identical with that of the electric ones with $\gamma=0$. If we set $\gamma=\frac{1}{2}$, the manners of action growth are similar for magnetic and electric EBI black holes, which implies electric and magnetic charges may contribute to action growth on equal footing for the dyonic EBI black hole. As we will see in the next subsection, this is indeed the case.

\subsection{Four-dimensional dyonic EBI black hole}

Since the integral (60) can not be integrated out for general $n$, for simplicity we only consider the $n=1$ case, i.e., the dyonic black hole in four dimensions.

For the dyonic EBI black hole with single horizon, bulk contributions to $\delta I$ are given by

$$
\begin{aligned}
I_{\mathcal{V}_{1}}-I_{\mathcal{V}_{2}}= & \frac{1}{16 \pi} \omega_{2} \delta t\left[\frac{2 \Lambda_{0}}{3} r_{+}^{3}+\beta^{2} \tilde{G}\left(r_{+}\right)-p^{2} \tilde{H}\left(r_{+}\right)\right. \\
& \left.-\beta^{2} \tilde{G}(0)+p^{2} \tilde{H}(0)\right] .
\end{aligned}
$$

with

$$
\begin{aligned}
& \tilde{G}(r) \equiv \int d r \sqrt{r^{4}+\frac{p^{2}+q^{2}}{\beta^{2}}}, \\
& \tilde{H}(r) \equiv \int d r \frac{1}{\sqrt{r^{4}+\frac{p^{2}+q^{2}}{\beta^{2}}}} .
\end{aligned}
$$


Action of the $r=0$ spacelike surface is

$$
I_{\mathcal{S}}=\frac{1}{16 \pi} \omega_{2} \delta t\left[3 \mu-\frac{\left(p^{2}+q^{2}\right)^{3 / 4} \sqrt{\beta}}{\sqrt{\pi}} \Gamma\left(\frac{1}{4}\right) \Gamma\left(\frac{5}{4}\right)\right] .
$$

The joint terms in action are

$$
\begin{aligned}
I_{\mathcal{B}^{\prime} \mathcal{B}}= & \frac{1}{16 \pi} \omega_{2} \delta t\left[\mu-\frac{2 \Lambda_{0}}{3} r_{+}^{3}+\frac{1}{2} \beta^{2} \tilde{G}\left(r_{+}\right)\right. \\
& \left.-\frac{\beta^{2} r_{+}}{2} \sqrt{r_{+}^{4}+\left(p^{2}+q^{2}\right) / \beta^{2}}\right] .
\end{aligned}
$$

Adding up all the contributions, the growth rate of action can be written as

$$
\frac{\delta I}{\delta t}=2 M-Q_{e} \Phi_{e}-\tilde{C},
$$

where

$$
\tilde{C}=\frac{\left(4 p^{2}+q^{2}\right) \omega_{2} \sqrt{\beta}}{48 \pi\left(q^{2}+p^{2}\right)^{1 / 4}} \frac{\Gamma\left(\frac{1}{4}\right) \Gamma\left(\frac{5}{4}\right)}{\Gamma\left(\frac{1}{2}\right)}
$$

and

$$
\begin{gathered}
M=\frac{\omega_{2}}{8 \pi} \mu, \quad Q_{e}=\frac{q}{16 \pi} \omega_{2}, \\
\Phi_{e}=\frac{q}{r_{+}}{ }_{2} F_{1}\left[\frac{1}{4}, \frac{1}{2}, \frac{5}{4},-\frac{p^{2}+q^{2}}{r_{+}^{4} \beta^{2}}\right] .
\end{gathered}
$$

are mass, electric charge and potential respectively.

The result (90) matches that of purely electric and magnetic EBI black holes with single horizon in general dimensions above, i.e., only the product of electric charge and potential appears in the expression of action growth, magnetic charge affects action growth only through some constant.

For the dyonic EBI black hole with inner and outer horizons, we have

$$
\begin{aligned}
I_{\mathcal{V}_{1}}-I_{\mathcal{V}_{2}}= & \left.\frac{1}{16 \pi} \omega_{2} \delta t\left[\frac{2 \Lambda_{0}}{3} r^{3}+\beta^{2} \tilde{G}(r)-p^{2} \tilde{H}(r)\right]\right|_{r_{-}} ^{r_{+}}, \\
I_{\mathcal{B}^{\prime} \mathcal{B}}+I_{\mathcal{C}^{\prime} \mathcal{C}}= & \frac{1}{16 \pi} \omega_{2} \delta t\left[\mu-\frac{2 \Lambda_{0}}{3} r^{3}+\frac{1}{2} \beta^{2} \tilde{G}(r)\right. \\
& \left.-\frac{\beta^{2} r}{2} \sqrt{r^{4}+\left(p^{2}+q^{2}\right) / \beta^{2}}\right]\left.\right|_{r_{-}} ^{r_{+}}
\end{aligned}
$$

Thus the growth rate of action is

$$
\frac{\delta I}{\delta t}=\left(M-Q_{e} \Phi_{e}\right)_{+}-\left(M-Q_{e} \Phi_{e}\right)_{-} .
$$

Similarly, only $Q_{e} \Phi_{e}$ appears in $\frac{\delta I}{\delta t}$. This result is formally identical with that of dyonic black holes in Einstein-Maxwell gravity. It seems that, independent of the linearity or nonlinearity of electromagnetic theory, only electric charge affects action growth of the black holes with double horizons, action growth rates vanish for the purely magnetically charged black holes.

After addition of the boundary term (70), for dyonic EBI black holes with single horizon, action growth becomes

$\frac{\delta I}{\delta t}=2 M-(1-\gamma) Q_{e} \Phi_{e}-\gamma Q_{m} \Phi_{m}-\tilde{C}_{1}$

where $Q_{m}, \Phi_{m}$ are magnetic charge and potential

$Q_{m}=\frac{p}{16 \pi} \omega_{2}, \quad \Phi_{m}=\frac{p}{r_{+}}{ }_{2} F_{1}\left[\frac{1}{4}, \frac{1}{2}, \frac{5}{4},-\frac{p^{2}+q^{2}}{r_{+}^{4} \beta^{2}}\right]$,

and

$$
\begin{aligned}
\tilde{C}_{1}= & \frac{\omega_{2} \sqrt{\beta}}{16 \pi^{3 / 2}\left(q^{2}+p^{2}\right)^{1 / 4}}\left[\frac{5}{6}\left(p^{2}+q^{2}\right)\right. \\
& \left.+\left(\frac{1}{2}-\gamma\right)\left(p^{2}-q^{2}\right)\right] \frac{\Gamma\left(\frac{1}{4}\right) \Gamma\left(\frac{5}{4}\right)}{\Gamma\left(\frac{1}{2}\right)} .
\end{aligned}
$$

Figure 6 shows $\frac{\delta I}{\delta t}$ versus $q$ and $\frac{\delta I}{\delta t}$ versus $p$. It is easy to see that, $\frac{\delta I}{\delta t}$ decreases with $q$ (or $p$ ) for fixed $p$ (or $q$ ), and Lloyd's bound is satisfied. Note that, on the left plot in Fig. 6, the lines with larger $\gamma$ are lower than the lines with smaller $\gamma$, while, on the right plot in Fig. 6, the lines with larger $\gamma$ are upper than the lines with smaller $\gamma$. This is because the coefficient before $Q_{e} \Phi_{e}$ in Eq. (96) is $(1-\gamma)$ while the coefficient before $Q_{m} \Phi_{m}$ in Eq. (96) is $\gamma$.

For dyonic BI black holes with double horizons, action growth becomes

$$
\begin{aligned}
\frac{\delta I}{\delta t}= & {\left[M-(1-\gamma) Q_{e} \Phi_{e}-\gamma Q_{m} \Phi_{m}\right]_{+} } \\
& -\left[M-(1-\gamma) Q_{e} \Phi_{e}-\gamma Q_{m} \Phi_{m}\right]_{-}
\end{aligned}
$$

Note from (96) and (99) that, if we set $\gamma=1, Q_{e} \Phi_{e}$ in the expressions of action growth vanishes, only $Q_{m} \Phi_{m}$ remains, electric charge affects action growth of the dyonic EBI black hole with single horizon only through some constant. If we set $\gamma=\frac{1}{2}$, the asymmetric part under $p \leftrightarrow q$ in (98) vanishes, then electric and magnetic charges contribute to action growth on equal footing for the four-dimensional dyonic EBI black holes. 

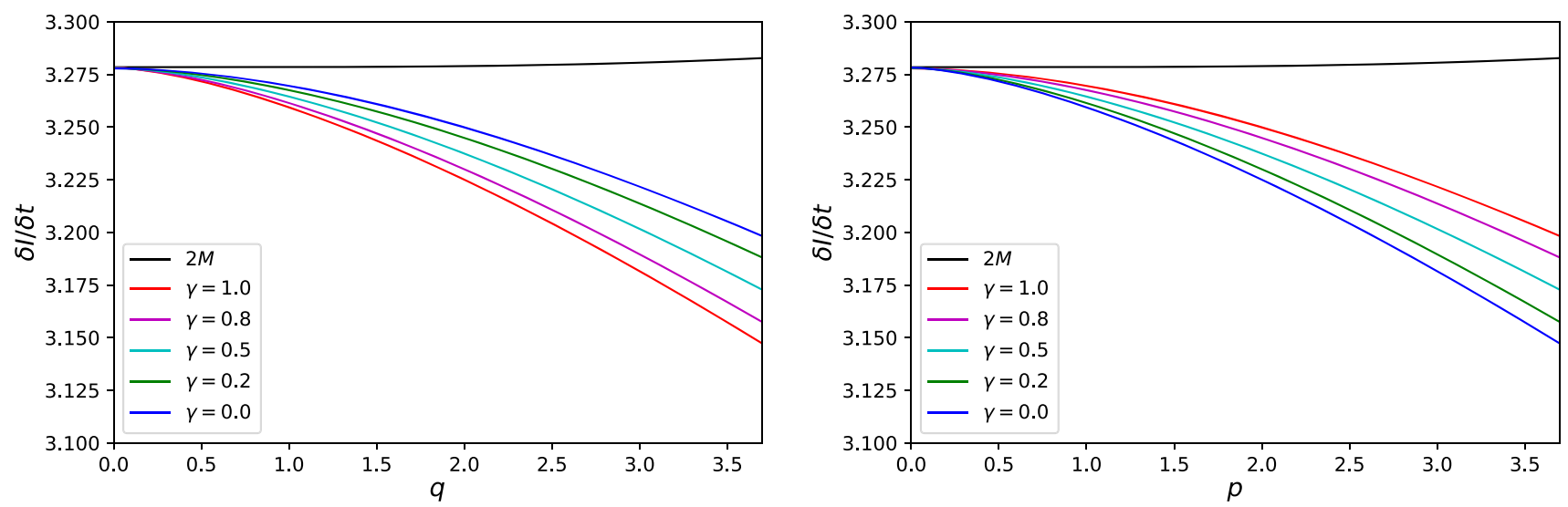

Fig. $6 \frac{\delta I}{\delta t}$ versus $q$ with fixed $p$ (left plot) and $\frac{\delta I}{\delta t}$ versus $p$ with fixed $q$ (right plot) for four-dimensional dyonic black hole with single horizon. The parameters are fixed as $\beta=0.15, r_{+}=5, \Lambda=-1, p=0.1$ for the left plot, and $\beta=0.15, r_{+}=5, \Lambda=-1, q=0.1$ for the right plot

\section{Summary and discussion}

In this paper, we study action growth of BI black holes. As a comparison, we first review action growth of dyonic black holes in Einstein-Maxwell gravity in general dimensions, and notice that similar to the four-dimensional case, action growth rates vanish for purely magnetic black holes if we don't consider the Maxwell boundary term. After the inclusion of Maxwell boundary term, if we set $\gamma=\frac{1}{2}$, then electric and magnetic charges contribute to action growth on equal footing, which is in accord with electric-magnetic duality. If we set $\gamma=1$, then action growth rates vanish for purely electric black holes.

We study action growth of electric BI black holes in massive gravity, and find that BI black holes in massive gravity always complexify faster than their Einstein gravity counterparts due to the back-reaction of graviton mass on geometry. If the BI boundary term is included, we find that action growth rates decrease as $\gamma$ increases.

For the EBI black holes, we first study action growths of the purely electric and magnetic black holes in general dimensions. Before considering the BI boundary term, action growths of electric and magnetic EBI black holes with single horizon are in different manners, which are $\frac{\delta I}{\delta t}=2 M-Q_{e} \Phi_{e}-\hat{C}$ and $\frac{\delta I}{\delta t}=2 M-\bar{C}$ respectively. We notice that, for the magnetic black holes, the constant $\bar{C}$ vanishes for even $n$. Therefore, in the dimensions with even $n$, action growth of magnetic EBI black holes saturates Lloyd's bound, i.e., it takes the identical form with that of the AdS-Schwarzschild black holes. In this case, magnetic charge affects action growth through back-reaction on the geometry. For EBI black holes with double hirizons, action growth of the electric ones takes the identical form with that of AdS-RN black holes, while action growth vanishes for the magnetic ones, this agrees with the result obtained from the dyonic black holes in Einstein-Maxwell gravity.
If we include the BI boundary term to the action, action growth of the electric (magnetic) EBI black holes with $\gamma=1$ takes similar form with that of the magnetic (electric) ones with $\gamma=0$. We find that, for electric EBI black holes with single horizon Lloyd's bound is satisfied, while, for the magnetic ones Lloyd's bound may be violated for some values of $\gamma$. Therefore, the inclusion of BI boundary term to action may lead to the violation of Lloyd's bound.

For the four-dimensional dyonic EBI black hole, calculation shows the manner of action growth of the black hole agrees with that of the purely electric and magnetic EBI black holes in general dimensions. When we set $\gamma=\frac{1}{2}$, electric and magnetic charges contribute to action growth on equal footing. Lloyd's bound is satisfied for the four-dimensional dyonic EBI black hole.

Acknowledgements KM would like to thank Profs. Peng Wang, Haitang Yang, Liu Zhao and Haishan Liu for valuable discussions.

Data Availability Statement This manuscript has no associated data or the data will not be deposited. [Authors' comment: This manuscript mainly involves theoretical analysis, there has not been experiment in this field as we know.]

Open Access This article is licensed under a Creative Commons Attribution 4.0 International License, which permits use, sharing, adaptation, distribution and reproduction in any medium or format, as long as you give appropriate credit to the original author(s) and the source, provide a link to the Creative Commons licence, and indicate if changes were made. The images or other third party material in this article are included in the article's Creative Commons licence, unless indicated otherwise in a credit line to the material. If material is not included in the article's Creative Commons licence and your intended use is not permitted by statutory regulation or exceeds the permitted use, you will need to obtain permission directly from the copyright holder.To view a copy of this licence, visit http://creativecomm ons.org/licenses/by/4.0/.

Funded by $\mathrm{SCOAP}^{3}$. 


\section{Appendix}

The magnetic potential is given by

$$
\begin{aligned}
\Phi_{m}= & \frac{\partial M}{\partial Q_{m}}=\frac{r_{+}^{2 n+1} \beta^{2} \omega_{2}^{n-1}}{2 n p}\left(\left(1+\frac{p^{2}}{r_{+}^{4} \beta^{2}}\right)^{n / 2}\right. \\
& \left.-{ }_{2} F_{1}\left[-\frac{1}{4}-\frac{n}{2},-\frac{n}{2}, \frac{3}{4}-\frac{n}{2},-\frac{p^{2}}{r_{+}^{4} \beta^{2}}\right]\right) .
\end{aligned}
$$

The two terms in Eq. (77) can be simplified to

$$
\begin{aligned}
& \frac{\omega_{2}^{n}}{16 \pi}\left(\frac{2 n+1}{2 n} \beta^{2} \bar{G}\left(r_{+}\right)-\frac{\beta^{2} r_{+}}{2 n} \sqrt{\left(r_{+}^{4}+p^{2} / \beta^{2}\right)^{n}}\right) \\
& \quad=-\frac{1}{n} Q_{m} \Phi_{m} .
\end{aligned}
$$

From the definition of $\bar{H}\left(r_{+}\right)$, we have

$$
\begin{aligned}
\bar{H}\left(r_{+}\right) & \equiv \int d r_{+}\left(r_{+}^{4}+\frac{p^{2}}{\beta^{2}}\right)^{n / 2-1} \\
& =\frac{r_{+}^{2 n-3}}{2 n-3} 2 F_{1}\left[\frac{3}{4}-\frac{n}{2}, 1-\frac{n}{2}, \frac{7}{4}-\frac{n}{2},-\frac{p^{2}}{r_{+}^{4} \beta^{2}}\right] .
\end{aligned}
$$

With the formula $2 F_{1}[a, b, c, z]=\frac{c-1}{(a-1)(b-1)} \frac{d}{d z} 2 F_{1}$ $[a-1, b-1, c-1, z], \bar{H}\left(r_{+}\right)$can be rewritten as

$$
\begin{aligned}
\bar{H}\left(r_{+}\right)= & -\frac{r_{+}^{2 n+1} \beta^{2}}{2 n p^{2}}\left(\left(1+\frac{p^{2}}{r_{+}^{4} \beta^{2}}\right)^{n / 2}\right. \\
& \left.-{ }_{2} F_{1}\left[-\frac{1}{4}-\frac{n}{2},-\frac{n}{2}, \frac{3}{4}-\frac{n}{2},-\frac{p^{2}}{r_{+}^{4} \beta^{2}}\right]\right) .
\end{aligned}
$$

Therefore

$$
\frac{\omega_{2}^{n}}{16 \pi} p^{2} \bar{H}\left(r_{+}\right)=-\frac{1}{n} Q_{m} \Phi_{m} .
$$

By comparison of Eqs. (101) and (104), we immediately have

$$
\begin{aligned}
& \frac{\omega_{2}^{n}}{16 \pi}\left(\frac{2 n+1}{2 n} \beta^{2} \bar{G}\left(r_{+}\right)\right. \\
& \left.-\frac{\beta^{2} r_{+}}{2 n} \sqrt{\left(r_{+}^{4}+p^{2} / \beta^{2}\right)^{n}}-p^{2} \bar{H}\left(r_{+}\right)\right)=0 .
\end{aligned}
$$

\section{References}

1. J. Erlich, E. Katz, D.T. Son, M.A. Stephanov, QCD and a holographic model of hadrons. Phys. Rev. Lett. 95, 261602 (2005). arXiv:hep-ph/0501128

2. I. Bredberg, C. Keeler, V. Lysov, A. Strominger, From NavierStokes To Einstein. JHEP 1207, 146 (2012). arXiv:1101.2451

3. G. Compere, P. McFadden, K. Skenderis, M. Taylor, The Holographic fluid dual to vacuum Einstein gravity. JHEP 1107, 050 (2011). arXiv:1103.3022

4. S.A. Hartnoll, C.P. Herzog, G.T. Horowitz, Building a holographic superconductor. Phys. Rev. Lett. 101, 031601 (2008). arXiv:0803.3295

5. S.A. Hartnoll, C.P. Herzog, G.T. Horowitz, Holographic superconductors. JHEP 0812, 015 (2008). arXiv:0810.1563

6. J. Maldacena, L. Susskind, Cool horizons for entangled black holes. Fortsch. Phys. 61, 781 (2013). arXiv:1306.0533

7. D. Stanford, L. Susskind, Complexity and shock wave geometries. Phys. Rev. D 90, 126007 (2014). arXiv:1406.2678

8. L. Susskind, Y. Zhao, Switchbacks and the Bridge to Nowhere. arXiv: 1408.2823

9. S. Karar, S. Gangopadhyay, Holographic complexity for Lifshitz system. Phys. Rev. D 98, 026029 (2018). arXiv:1711.10887

10. B. Chen, W.M. Li, R.Q. Yang, C.Y. Zhang, S.J. Zhang, Holographic subregion complexity under a thermal quench. JHEP 1807, 034 (2018). arXiv:1803.06680

11. A. Bhattacharya, S. Roy, Holographic Entanglement Entropy, Subregion Complexity and Fisher Information metric of 'black' NonSUSY D3 Brane. arXiv:1807.06361

12. L.P. Du, S.F. Wu, H.B. Zeng, Holographic complexity of the disk subregion in (2+1)-dimensional gapped systems. Phys. Rev. D 98, 066005 (2018). arXiv: 1803.08627

13. S.J. Zhang, Subregion complexity and confinement-deconfinement transition in a holographic QCD model. arXiv:1808.08719

14. Y. Ling, Y. Liu, C.Y. Zhang, Holographic subregion complexity in Einstein-Born-Infeld theory. arXiv:1808.10169

15. A.R. Brown, D.A. Roberts, L. Susskind, B. Swingle, Y. Zhao, Complexity equals action. Phys. Rev. Lett. 116, 191301 (2015). arXiv: 1509.07876

16. A.R. Brown, D.A. Roberts, L. Susskind, B. Swingle, Y. Zhao, Complexity, action, and black holes. Phys. Rev. D 93, 086006 (2016). arXiv:1512.04993

17. G.'t Hooft, Dimensional reduction in quantum gravity. Conf. Proc. C 930308, 284 (1993). arXiv:gr-qc/9310026

18. L. Susskind, The world as a hologram. J. Math. Phys. 36, 6377 (1995). arXiv:hep-th/9409089

19. J.D. Bekenstein, A universal upper bound on the entropy to energy ratio for bounded systems. Phys. Rev. D 23, 287 (1981)

20. N. Margolus, L.B. Levitin, The maximum speed of dynamical evolution. Phys. D 120, 188 (1998). arXiv:quant-ph/9710043

21. S. Lloyd, Ultimate physical limits to computation. Nature 406(6799), 1047 (2000)

22. R.G. Cai, S.M. Ruan, S.J. Wang, R.Q. Yang, R.H. Peng, Action growth for AdS black holes. JHEP 1609, 161 (2016). arXiv: 1606.08307

23. R.G. Cai, M. Sasaki, S.J. Wang, Action growth of charged black holes with a single horizon. Phys. Rev. D 95, 124002 (2017). arXiv: 1702.06766

24. J. Tao, P. Wang, H. Yang, Testing holographic conjectures of complexity with Born-Infeld black holes. Eur. Phys. J. C 77(12), 817 (2017). arXiv:1703.06297

25. P. Wang, H. Yang, S. Ying, Action growth in $f(R)$ gravity. Phys. Rev. D 96(4), 046007 (2017). arXiv:1703.10006 
26. B. Swingle, Y. Wang, Holographic complexity of EinsteinMaxwell-Dilaton gravity. JHEP 1809, 106 (2018). arXiv: 1712.09826

27. W.D. Guo, S.W. Wei, Y.Y. Li, Y.X. Liu, Complexity growth rates for AdS black holes in massive gravity and $f(R)$ gravity. Eur. Phys. J. C 77(12), 904 (2017). arXiv:1703.10468

28. W.J. Pan, Y.C. Huang, Holographic complexity and action growth in massive gravities. Phys. Rev. D 95, 126013 (2017). arXiv: 1612.03627

29. Y.S. An, R.H. Peng, The effect of Dilaton on the holographic complexity growth. Phys. Rev. D 97, 066022 (2018). arXiv:1801.03638

30. R. Auzzi, S. Baiguera, M. Grassi, G. Nardelli, N. Zenoni, Complexity and action for warped AdS black holes. JHEP 1809, 013 (2018). arXiv:1806.06216

31. M. Alishahiha, A.F. Astaneh, A. Naseh, M.H. Vahidinia, On complexity for higher derivative gravities. JHEP 1705, 009 (2017). arXiv: 1702.06796

32. J. Jiang, H. Zhang, Surface term, corner term, and action growth in $\mathrm{F}($ Riemann) gravity theory. arXiv: 1806.10312

33. A. Reynolds, S.F. Ross, Complexity in de Sitter Space. Class. Quant. Grav. 34(17), 175013 (2017). arXiv:1706.03788

34. P.A. Cano, R.A. Hennigar, H. Marrochio, Complexity growth rate in Lovelock gravity. Phys. Rev. Lett. 121, 121602 (2018). arXiv: 1803.02795

35. L. Sebastiani, L. Vanzo, S. Zerbini, Action growth for black holes in modified gravity. Phys. Rev. D 97(4), 044009 (2018). arXiv: 1710.05686

36. S. Mahapatra, P. Roy, On the time dependence of holographic complexity in a dynamical Einstein-dilaton model. arXiv:1808.09917

37. R.Q. Yang, Strong energy condition and complexity growth bound in holography. Phys. Rev. D 95(8), 086017 (2017). arXiv: 1610.05090

38. S. Chapman, H. Marrochio, R.C. Myers, Holographic complexity in Vaidya spacetimes I. JHEP 1806, 046 (2018). arXiv: 1804.07410

39. S. Chapman, H. Marrochio, R.C. Myers, Holographic complexity in Vaidya spacetimes II. JHEP 1806, 114 (2018). arXiv: 1805.07262

40. J. Jiang, Action growth rate for a higher curvature gravitational theory. arXiv: 1810.00758
41. Y.G. Miao, L. Zhao, Complexity-action duality of the shock wave geometry in a massive gravity theory. Phys. Rev. D 97(2), 024035 (2018). arXiv: 1708.01779

42. S.A.H. Mansoori, V. Jahnke, M.M. Qaemmaqami, Y.D. Olivas, Action growth rate for a higher curvature gravitational theory. arXiv: 1808.00067

43. D.C. Zou, S.J. Zhang, B. Wang, Critical behavior of Born-Infeld AdS black holes in the extended phase space thermodynamics. Phys. Rev. D 89(4), 044002 (2014). arXiv:1311.7299

44. K. Meng, D.B. Yang, Black holes of dimensionally continued gravity coupled to Born-Infeld electromagnetic field. Phys. Lett. B 780, 363 (2018). arXiv: 1712.08798

45. K. Meng, Hairy black holes of Lovelock-Born-Infeld-scalar gravity. Phys. Lett. B 784, 56 (2018). arXiv:1804.10951

46. K. Goto, H. Marrochio, R.C. Myers, L. Queimada, B. Yoshida, Holographic complexity equals which action? JHEP 1902, 160 (2019). arXiv: 1901.00014

47. H.S. Liu, H. Lu, "Action Growth of Dyonic Black Holes and Electromagnetic Duality," arXiv:1905.06409

48. J. Jiang, M. Zhang, Holographic complexity of the electromagnetic black hole. arXiv: 1905.07576

49. S.H. Hendi, B.E. Panah, S. Panahiyan, Einstein-Born-Infeldmassive gravity: adS-Black hole solutions and their thermodynamical properties. JHEP 1511, 157 (2015). arXiv:1508.01311

50. L. Lehner, R.C. Myers, E. Poisson, R.D. Sorkin, Gravitational action with null boundaries. Phys. Rev. D 94, 084046 (2016). arXiv: 1609.00207

51. D. Brill, G. Hayward, Is the gravitational action additive? Phys. Rev. D 50, 4914 (1994). arXiv:gr-qc/9403018

52. R.G. Cai, Y.P. Hu, Q.Y. Pan, Y.L. Zhang, Thermodynamics of Black Holes in massive gravity. Phys. Rev. D 91(2), 024032 (2015). arXiv: 1409.2369

53. S. Li, H. Lu, H. Wei, Dyonic (A)dS Black Holes in EinsteinBorn-Infeld theory in diverse dimensions. JHEP 1607, 004 (2016). arXiv:1606.02733 